\title{
Leonurine alleviates ferroptosis in cisplatin-induced acute kidney injury by activating the Nrf2 signaling pathway
}

\author{
Jianqiang $\mathrm{HU}^{1}$, Wenjing $\mathrm{Gu}^{1}$, Ning $\mathrm{Ma}^{1}$, Xiaoye $\mathrm{Fan}^{2}$, and Xinxin $\mathrm{Ci}^{1}$ \\ ${ }^{1}$ Jilin University First Hospital \\ ${ }^{2}$ Institute of Translational Medicine, The First Hospital, Jilin University
}

October 28, 2021

\begin{abstract}
Background and purpose: Increasing evidence suggests that ferroptosis plays a key role in the pathophysiology of acute kidney injury induced by cisplatin. The Nrf2 signaling pathway regulates oxidative stress and lipid peroxidation and positively regulates cisplatin-induced AKI (CI-AKI). However, Nrf2 and its activator leonurine on ferroptosis after CI-AKI remain unclear. Experimental Approach: The anti-ferroptotic effects of Nrf2 and its activator leonurine were assessed using a mouse model of cisplatin-induced AKI. In vitro, the potential effects of leonurine on erastin- and RSL3-induced HK-2 human PTEC ferroptosis were examined. Key Results: As expected, Nrf2 deletion induced ferroptosis-related protein expression and iron accumulation in vivo, further aggravating CI-AKI. The Nrf2 activator leonurine prevented iron accumulation and lipid peroxidation and inhibited ferroptosis in vitro, while these effects were abolished in siNrf2-treated cells. Moreover, leonurine potently ameliorated cisplatin-induced renal damage, as indicated by the assessment of SCr, BUN, KIM-1, and NGAL. Importantly, leonurine activated the Nrf2 antioxidative signaling pathway and prohibited changes in ferroptosis-related morphological and biochemical indicators, such as the MDA level, SOD and GSH depletion and GPX4 and xCT downregulation, in CI-AKI. Moreover, Nrf2 $\mathrm{KO}$ mice were more susceptible to ferroptosis after CI-AKI than control mice, and the protective effects of leonurine on AKI and ferroptosis were largely abolished in Nrf2 KO mice. Conclusion and Implications: These data suggest that the renal protective effects of Nrf2 and its activator leonurine on CI-AKI are achieved at least partially by inhibiting lipid peroxide-mediated ferroptosis and highlight the potential of leonurine as a CI-AKI treatment.
\end{abstract}

Leonurine alleviates ferroptosis in cisplatin-induced acute kidney injury by activating the Nrf2 signaling pathway

Jianqiang $\mathrm{Hu}{ }^{\mathrm{a} \#}$, Wenjing $\mathrm{Gu}{ }^{\mathrm{b} \#}$, Ning Ma ${ }^{\mathrm{a}}$, Xiaoye Fan ${ }^{\mathrm{a}}$, Xinxin $\mathrm{Ci}^{\mathrm{a}^{*}}$

a Institute of Translational Medicine, The First Hospital of Jilin University, Changchun, Jilin, 130001, China

b Department of Otolaryngology Head and Neck Surgery, The First Hospital of Jilin University, Changchun, Jilin 130001, China

\# Jianqiang $\mathrm{Hu}$ and Wenjing Gu contributed equally to this work.

*corresponding author.

E-mail address: cixinxin@jlu.edu.cn

Full postal address: Dongminzhu road 519, The First Hospital of Jilin University, Changchun, Jilin, 130001, China.

Data Availability Statement 
The data that support the findings of this study are available from the corresponding author upon reasonable request. Some data may not be made available becaused of privacy or ethical restrictions.

\begin{abstract}
Background and purpose: Increasing evidence suggests that ferroptosis plays a key role in the pathophysiology of acute kidney injury (AKI) induced by cisplatin. The Nrf2 signaling pathway regulates oxidative stress and lipid peroxidation and positively regulates cisplatin-induced AKI (CI-AKI). However, its effect as well as that of its activator leonurine on ferroptosis after CI-AKI remain unclear.
\end{abstract}

Experimental Approach: The anti-ferroptotic effects of Nrf2 and its activator leonurine were assessed using a mouse model of cisplatin-induced AKI. In vitro, the potential effects of leonurine on erastin- and RSL3induced HK-2 human PTEC ferroptosis were examined.

Key Results: As expected, Nrf2 deletion induced ferroptosis-related protein expression and iron accumulation in vivo, further aggravating CI-AKI. The Nrf2 activator leonurine prevented iron accumulation and lipid peroxidation and inhibited ferroptosis in vitro, while these effects were abolished in siNrf2-treated cells. Moreover, leonurine potently ameliorated cisplatin-induced renal damage, as indicated by the assessment of SCr, BUN, KIM-1, and NGAL. Importantly, leonurine activated the Nrf2 antioxidative signaling pathway and prohibited changes in ferroptosis-related morphological and biochemical indicators, such as the MDA level, SOD and GSH depletion and GPX4 and xCT downregulation, in CI-AKI. Moreover, Nrf2 KO mice were more susceptible to ferroptosis after CI-AKI than control mice, and the protective effects of leonurine on AKI and ferroptosis were largely abolished in Nrf2 KO mice.

Conclusion and Implications: These data suggest that the renal protective effects of Nrf2 and its activator leonurine on CI-AKI are achieved at least partially by inhibiting lipid peroxide-mediated ferroptosis and highlight the potential of leonurine as a CI-AKI treatment.

Key words : Cisplatin-induced acute kidney injury; Ferroptosis; Nrf2; Leonurine

Abbreviations: Leo, leonurine; CDDP, cisplatin; AKI, acute kidney injury; CI-AKI, cisplatin-induced acute kidney injury; HK-2, Human Kidney-2; SCr, serum creatinine; BUN, Blood Urea Nitrogen; SOD, Superoxide Dismutase; MDA, malondialdehyde; GSH, glutathione; Nrf2, nuclear factor erythroid-2; HO-1, Heme Oxygenase-1; NQO1, NAD(P)H quinone dehydrogenase 1; KIM1, kidney injury molecule-1; NGAL, Neutrophil gelatinase-associated lipocalin; FTH-1, ferritin heavy chain; FTL, ferritin light chain; TFR, transferrin receptor 1; GPX4, glutathione peroxidase 4; XCT, cystine/glutamateantiporter system; SLC7A11, Solute Carrier Family 7 Member 11; ROS, reactive oxygen species; LPO, lipid peroxidation; RSL3:(1S,3R)RSL3; CCK-8, Cell Counting Kit-8; FBS, fetal bovine serum; DCFH-DA, 2,7-Dichlorodihydrofluorescein diacetate; PBS, phosphate buffered saline; DMSO, Dimethyl sulfoxide; HE, Hematoxylin and eosin staining; PCR, Polymerase Chain Reaction; UUO, unilateral ureteral obstruction.

\title{
Introduction
}

Acute kidney injury (AKI), a common and severe syndrome characterized by tubular damage and sudden loss of renal function within hours, is a global health problem with high morbidity and mortality(Hoste et al., 2018). As an antitumor drug, cisplatin is widely used in the treatment of solid tumors and is one of the main causes of AKI in patients with cancer(Yang et al., 2018). Because the exact molecular mechanisms of CI-AKI are not fully understood, strategies for the prevention and treatment of AKI are limited. Tubular cell injury and death play vital roles in the initiation and progression of AKI(Deng et al., 2021; Linkermann et al., 2014). Previous studies focused on the induction of renal tubular cell apoptosis and necrosis by cisplatin, but specific inhibitors of both apoptosis and necrosis failed to completely prevent or stop CI-AKI(Herzog et al., 2012; Tristao et al., 2012). Ferroptosis, a new type of "regulated cell death", was recently reported to be involved in the pathological processes of cisplatin-induced AKI (Hu et al., 2020). Thus, elucidating the mechanisms by which cisplatin induces ferroptosis will have important clinical value for alleviating AKI.

In contrast to other regulatory cell death mechanisms, such as apoptosis, necrosis and pyrogen death, fer- 
roptosis is a newly discovered regulatory type of cell death caused by iron-dependent lipid peroxidation (Stockwell et al., 2017) (Deng et al., 2021). In the case of ferroptosis, low FTH-1 expression and TFR overexpression lead to excessive iron accumulation, which in turn promotes ROS production via Fenton reactions (Dixon and Stockwell, 2014). At the same time, the function of system XC is disrupted during ferroptosis, thereby decreasing the production of glutathione (GSH) and inhibiting the antioxidant capacity of GPX4 (Wang et al., 2020) (Maiorino et al., 2018). Thus, ROS have been demonstrated to a central role in the entire ferroptosis process (Park and Chung, 2019). In cisplatin-induced AKI, the generation of excess ROS can cause oxidative stress and lead to tubular cell damage. Nrf2, the most important nuclear transcription factor for antioxidative stress, regulates not only the expression of a series of signaling proteins and enzymes to maintain cellular redox homeostasis but also many important genes related to iron storage and transport (Dodson et al., 2019). Previous studies have highlighted the role of Nrf2 in alleviating lipid peroxidation and ferroptosis and further elucidated the role of the Nrf2-lipid peroxidation-ferroptosis axis in neurodegenerative and cardiovascular diseases(Dodson et al., 2019). Many Nrf2 activators have been reported to alleviate cisplatin-induced nephrotoxicity by inhibiting ROS generation (Fan et al., 2020). In addition, ferrostatin-1, the most potent inhibitor of ferroptosis, was shown to significantly reduce cisplatin-induced HK-2 cell death and attenuate CI-AKI in mice(Deng et al., 2019). Considering the close relationship among ROS, Nrf2 and ferroptosis, we proposed that controlling ferroptosis via Nrf2 activation might improve CI-AKI.

Leonurine, a major active alkaloid compound found only in motherwort, has been successfully extracted and depurated $^{(\mathrm{Li} \text { et al., } 2020)}$. Leonurine was recently shown to regulate various pathological processes, including oxidative stress, inflammation, fibrosis, and apoptosis, as well as a variety of metabolic disorders. Leonurine protects against LPS-induced acute renal injury and fibrosis induced by unilateral ureteral obstruction (UUO) in mice(Cheng et al., 2015). However, an alleviating effect of leonurine on CI-AKI has not been reported. At the same time, some studies have shown that leonurine can alleviate aging and ischemic stroke in mice by activating the Nrf2 pathway(Chen et al., 2019; Xie et al., 2019), but its effect on ferroptosis has not been investigated. Herein, we first investigated whether the levels of Nrf2 are directly related to ferroptosis sensitivity in mice with CI-AKI. We then investigated the potential of leonurine to protect against ferroptosis in vitro and in vivo. Finally, using Nrf2 siRNA and Nrf2 knockout (KO) mice, we further assessed the underlying mechanisms by which leonurine protects against ferroptosis and AKI in vitro and in vivo.

\section{Methods}

\subsection{Materials}

Leonurine (98.24\%) was purchased from PuFeiDe Biotech Co. (Chengdu, China). Erastin and RSL3 were purchased from Selleckchem (Shanghai, China). Cisplatin was purchased from Med Chem Express (New Jersey, USA). Primary antibodies against $\beta$-actin, KIM1, NGAL, GPX4, XCT, FTL, FTH-1, TFR, Nrf2, HO-1, and NQO1 were purchased from Abcam (Cambridge MA, USA), Cell Signaling Technology (Danvers, Massachusetts, USA), R\&D Systems (Minnesota, USA) and Proteintech (Wuhan, China). The BCA protein assay kit was purchased from Thermo Fisher Scientific (MA, USA). The iron assay kit was purchased from BioAssay Systems (Northern California Bay Area, USA). Calcein-acetoxymethyl (AM) was purchased from Beyotime Biotechnology (Jiangsu, China). DMEM/F-12, penicillin, streptomycin and trypsin EDTA, used for cell culturing, were purchased from hyclone (Logan, Utah, USA). Dimethyl sulfoxide (DMSO) and DCFHDA were purchased from Sigma (St. Louis, Missouri, USA). Cell Counting Kit-8 (CCK-8) was purchased from Bimake (Houston, USA). Nrf2 siRNA was obtained from Hanbio Biotechnology Co., Ltd. (Shanghai, China). SYBR Green I was purchased from Roche (Basel, Switzerland).

\subsection{Cell treatment}

HK-2 cells were cultured in DMEM/F12 supplemented with $10 \%$ fetal bovine serum (FBS) and $100 \mathrm{U} / \mathrm{ml}$ penicillin/streptomycin at 37 and $5 \% \mathrm{CO}_{2}$. After being cultured in the absence or presence of different concentrations of leonurine for $1 \mathrm{~h}$, the cells were treated with different concentrations of RSL3 or erastin for $24 \mathrm{~h}$ and then collected or stained for subsequent experiments.

\subsection{Cell viability}


Cell viability was determined using the CCK-8 assay. HK-2 cells were inoculated into 96 -well culture plates $\left(1 \times 10^{4} /\right.$ well $)$ and treated with leonurine and cisplatin for $24 \mathrm{~h}$. Thereafter, $10 \mu \mathrm{L}$ of CCK- 8 reagent was added to each well, and the plate was cultured at 37 for $1 \mathrm{~h}$. The OD value at $450 \mathrm{~nm}$ was then measured with an enzyme-labeling instrument to determine the cell survival rate.

\subsection{Analysis of intracellular ROS levels}

After planking and drug treatment for $24 \mathrm{~h}$, the cells were mixed with DCFH-DA $(1 \mu \mathrm{M})$ and incubated in the dark at room temperature for 30 minutes. After washing the cells with PBS three times, they were observed by a fluorescence microscope. The OD value was determined by an enzyme-labeling instrument, and the ROS content was determined.

\subsection{Analysis of lipid peroxidation (LPO)}

A Liperfluo assay kit was used to detect the lipid peroxide content in HK-2 cells. After washing three times, the cells were mixed with $1 \mu \mathrm{M}$ Liperfluo, incubated in the dark at room temperature for 30 minutes, and then washed with PBS three times. Lipid peroxide was finally measured and evaluated by confocal microscopy.

\subsection{Animal experiments}

Female C57BL/6 mice (8-10 weeks, 20-22g) and Nrf2 KO mice were purchased from Vital River (Beijing, China) and Jackson Laboratory (Bar, Harbor, USA), respectively. After one week of acclimatization, CI-AKI was established in female C57BL/6 mice. The experimental animals were divided into five groups as follows ( $\mathrm{n}=10$ /group): control (0.1\% DMSO in normal saline), cisplatin $(20 \mathrm{mg} / \mathrm{kg})$, cisplatin $(20 \mathrm{mg} / \mathrm{kg})+$ leonurine $(50 \mathrm{mg} / \mathrm{kg}$ and $100 \mathrm{mg} / \mathrm{kg})$ and leonurine only $(100 \mathrm{mg} / \mathrm{kg}$ dissolved in $0.1 \%$ DMSO). After fasting, the mice in the cisplatin $(20 \mathrm{mg} / \mathrm{kg})$ + leonurine group were treated with $50 \mathrm{mg} / \mathrm{kg}$ and $100 \mathrm{mg} / \mathrm{kg}$ leonurine by gavage and intraperitoneally injected with cisplatin $(20 \mathrm{mg} / \mathrm{kg})$ one hour later to induce acute renal injury. Mice in the control, cisplatin and leonurine groups were treated with $0.1 \%$ DMSO in normal saline, cisplatin $(20 \mathrm{mg} / \mathrm{kg})$ and leonurine $(100 \mathrm{mg} / \mathrm{kg}$ dissolved in $0.1 \% \mathrm{DMSO})$, respectively. On the second and third days, the mice in the cisplatin $(20 \mathrm{mg} / \mathrm{kg})+$ leonurine and leonurine only groups were administered leonurine by gavage once more. On the fourth day, all the mice were sacrificed, and kidney and blood samples were collected for further experiments. The mice were housed in a controlled living environment and provided adequate amounts of food and water.

\subsection{Hematoxylin and eosin staining (HE)}

Renal tissue portions were fixed overnight with $4 \%$ paraformaldehyde, embedded in paraffin, sectioned, stained with HE, and then observed under an optical microscope (Olympus, Japan). The degree of renal injury was evaluated in three random fields per sample by grading the renal tissue injury as follows: 0-10\%, $0 ; 11-25 \%, 1 ; 26-50 \%, 2 ; 51-75 \%, 3$; and $76-100 \%, 4$. At least five renal regions on each slide were randomly selected.

\subsection{Immunohistochemistry and enhanced Prussian blue staining}

For immunohistochemistry analysis, the slides were sequentially incubated with $5 \%$ bovine serum albumin for $1 \mathrm{~h}$, with a primary antibody targeting F4/80 overnight, and then with the appropriate secondary antibody. After incubation with the secondary antibody, the cells were observed under an optical microscope. Enhanced Prussian blue staining was used to detect the iron deposition in renal tissue. Mouse kidney tissues were fixed with $3 \%$ glutaraldehyde overnight, washed three times, and sectioned at a thickness of $4 \mu \mathrm{m}$ for prussian blue staining. After washing with PBS, nuclei were stained with hematoxylin, and the iron deposition in the tissues was finally observed under an optical microscope.

\subsection{Transmission electron microscopy}

Morphological changes in renal tissue mitochondria were observed by electron microscopy. In brief, the tissues were fixed with pentanediol, sectioned with an ultrathin microtome, dehydrated, and stained many 
times. Images were then obtained with a transmission electron microscope, and more than 5 different visual fields from each slice were observed.

\subsection{RNA isolation and real-time PCR}

Total RNA was extracted from fresh renal tissue by using the TRIzol method and then reverse transcribed into cDNA. The cDNA content was detected by real-time quantitative PCR to determine the gene transcription levels in the tissue. Samples from five independent experiments were utilized, and each sample was subjected to real-time PCR in triplicate. The primers used in this study are provided in Table S1.

\subsection{Assays of renal functional parameters as well as GSH, MDA and SOD}

The levels of BUN and SCr, which can indicate the level of renal function, were detected in serum samples, whereas the levels of GSH, MDA and SOD were detected in renal tissue and cell suspensions. The above mentioned indices were detected by BUN, SCr, GSH, MDA and SOD assay kits according to the manufacturers' instructions.

\subsection{Iron content analysis}

The intracellular iron level was measured by a calcein-AM kit. After cell plating and drug treatment for 24 h, HK-2 cells were treated with AM $(2 \mu \mathrm{M})$ in the dark at 37 for 30 minutes. Next, the cells were washed and analyzed with a fluorescence microscope. To perform fluorescence imaging of $\mathrm{Fe}^{2+}$ in living cells, HK-2 cells were inoculated into confocal dishes and cultured overnight at 37 in a $5 \% \mathrm{CO}_{2}$ incubator. FerroOrange working solution $(1 \mu \mathrm{M})$, purchased from Dojindo (Kumamoto, Kyushu, Japan), was added to the cells, which were cultured in a $5 \% \mathrm{CO}_{2}$ incubator at 37 and then observed by a fluorescence confocal microscope. In vivo, an iron assay kit was used to detect the iron ion content in mouse serum, which was determined by detecting the absorbance at $405 \mathrm{~nm}$.

\subsection{Western blot assay}

Fresh cells or kidney tissues were collected and lysed on ice for 30 minutes. Equal amounts of protein $(20 \mu \mathrm{g})$ were separated by 10-15\% SDS-PAGE, and the separated proteins were transferred onto PVDF membranes and blocked with $5 \%$ nonfat powder milk in PBS buffer for $1 \mathrm{~h}$ at room temperature. The cells were incubated with primary antibodies against $\beta$-actin, KIM1, NGAL, GPx4, xCT, FTL, FTH-1, TFR, Nrf2, HO-1 and NQO1 overnight at $4^{\circ} \mathrm{C}$ and then with an HRP-conjugated secondary antibody (1:5000) at room temperature for $1 \mathrm{~h}$. The relative expression levels were determined with Image $\mathrm{J}$ software.

\subsection{RNA interference}

HK-2 cells were transfected with Nrf2 siRNAs for $72 \mathrm{~h}$, after which Western blotting was performed with an anti-Nrf2 antibody (1:1000) to assess their transfection efficiencies. The siRNA with the highest transfection efficiency was selected for the cell treatments described above and for subsequent cell experiments.

\subsection{Statistical analysis}

The data were statistically analyzed by SPSS 19.0 (IBM) and are expressed as the means \pm SEMs. The experimental data were compared by one-way analysis of variance (ANOVA). A P value $<0.05$ was considered statistically significant. The sample size needed for statistical analysis was determined to be at least 5 animals in each group ( $\mathrm{N}=5$, where $\mathrm{n}$ refers to the number of independent data points).

\section{Results}

\subsection{Nrf2 KO aggravates cisplatin-induced kidney injury and iron accumulation}

Nrf2 and its regulatory genes are reported to be involved in preventing the progression of ferroptosis, but their regulatory effect on ferroptosis in CI-AKI has not been clarified. As shown in Fig. 1A-D, Nrf2 KO aggravated cisplatin-induced kidney injury, which was characterized by elevated levels of Bun and SCr as well as more morphological damage, including necrosis, brush edge loss, cast formation, tubular degeneration, and vacuolization. Nrf2 targets play key roles in mediating iron metabolism, which is important for the 
initiation of ferroptosis. Therefore, we investigated the changes in the iron levels in Nrf2 KO mice and found that cisplatin injection increased the iron levels in Nrf2 KO mice compared with wild-type mice (Fig. 1E). In addition, lower expression levels of the antioxidant response genes Nrf2, HO-1 and NQO1 were observed in Nrf2 KO mice compared with wild-type mice. Interestingly, loss of Nrf2 not only increased TFR expression but also led to a compensatory increase in FTH-1 and FTL (Fig. 1F-H).

\subsection{Nrf2 KO mice are susceptible to cisplatin-induced lipid peroxidation and ferroptosis}

Nrf2 is a key reliever of lipid peroxidation and ferroptosis, and abnormal Nrf2 signaling is thought to aggravate the disease by regulating lipid peroxides and ferroptosis. We investigated lipid peroxidation and ferroptosis in Nrf2 KO mice and found that their malondialdehyde (MDA) levels were increased to a greater extent than those in the WT mice, while the GSH levels were significantly decreased in Nrf2 KO mice after cisplatin injection compared to those in the WT mice (Fig. 2A-B). We further examined the protein expression of GPX4 and xCT, biomarkers of ferroptosis, to clarify the role of Nrf2 in ferroptosis. As shown in Fig. 2C-F, both the mRNA and protein levels of GPX4 and xCT were significantly decreased in Nrf2 KO mice compared with WT mice.

\subsection{Leonurine inhibits iron accumulation and ferroptosis in HK-2 cells}

HK-2 cells were treated with different doses of erastin or RSL3, classical inducers of ferroptosis, to determine the exact concentrations that inhibited cell growth. As shown in Fig. 3A and 3B, erastin $(30 \mu \mathrm{M})$ and RSL3 $(0.4 \mu \mathrm{M})$ inhibited cell viability in a dose-dependent manner. Importantly, leonurine treatment significantly ameliorated the effects of erastin and RSL3 on cell death (Fig. 3C-D). Then, we used a fluorescent probe calcein-AM, to detect changes in cellular labile iron in cells based on its ability to be rapidly quenched when bound to cellular iron. As shown in Fig. E, RSL3 treatment markedly decreased the fluorescence signals, indicating that cellular iron levels were increased by RSL3 treatment but reversed by leonurine treatment. We next detected $\mathrm{Fe}^{2+}$ in HK-2 cells using FerroOrange. As shown in Fig. 3F, the $\mathrm{Fe}^{2+}$ content was increased after RSL3 treatment but decreased after leonurine treatment. We further evaluated the protein expression of key factors regulating cellular iron homeostasis. As shown in Figure 3G and 3H, RSL3 significantly increased the protein levels of TFR, FTH-1 and FTL, and these trends were reversed by leonurine treatment.

\subsection{Leonurine inhibited ROS generation and lipid peroxidation and restored the expression of ferroptosis-related genes}

Free iron can interact with ROS to form lipid radicals that rapidly react with oxygen to induce lipid peroxidation. We first investigated intracellular ROS using the sensitive fluorescent probe DCFH-DA, revealing that ROS generation was induced by RSL3 but reversed by leonurine (Fig. 4A). In addition, we used the Liperfluo probe to detect lipid peroxides and found that lipid peroxidation was increased by RSL3 treatment but decreased by leonurine (Fig. 4B). To further confirm that the leonurine-regulated ferroptosis blockade was associated with lipid peroxidation, we examined the cellular MDA and GSH levels. RSL3 treatment significantly downregulated GSH and upregulated MDA, and these trends were reversed by leonurine treatment (Fig. 4C to 4D). Next, we further evaluated the regulatory effect of leonurine on the expression of antioxidant and ferroptosis-related target molecules. RSL3 treatment significantly decreased the GPX4 and $\mathrm{xCT}$ expression compared to that in the control group, but leonurine restored these changes in a dosedependent manner (Fig. 4E-F). Moreover, leonurine dose-dependently upregulated the protein expression of Nrf2, NQO1 and HO-1 (Fig. 4G).

\subsection{Knockdown Nrf2 abolishes the effect of leonurine on ROS generation, lipid peroxidation and ferroptosis}

Considering the role of Nrf2 in preventing lipid peroxidation and ferroptosis, we attempted to investigate the potential for Nrf2 to mediate the protective effect of leonurine on ferroptosis. Control or Nrf2 siRNA was transiently transfected into cells, and Nrf2 protein expression was shown to be significantly inhibited in the Nrf2 siRNA group (Fig. 5A-B). As shown in Fig. 5C, the protective effect of leonurine on RSL3-induced cell death was alleviated in Nrf2 knockdown cells. Furthermore, the protective effects of leonurine against 
ROS generation, MDA production and GSH depletion were nearly abolished in Nrf2 knockdown cells (Fig. 5D-F). The inhibitory effect of leonurine on cellular iron levels was almost eliminated in Nrf2 knockdown cells as determined by calcein-AM detection (Fig. 5G). Next, we examined the protein expression of key factors regulating cellular iron homeostasis and ferroptosis. As shown in Fig. 5H-J, the regulatory effects of leonurine on TFR, FTH1, GPX4 and XCT are nearly eliminated in Nrf2 knockdown cells.

\subsection{Leonurine protects against CI-AKI}

Based on the effect of leonurine on ferroptosis in vitro, we further evaluated its potential effects on CI-AKI in vivo. Compared to those in the control group, the $\mathrm{SCr}$ and BUN levels in mice receiving the cisplatin injection were significantly increased; however, the levels were reversed by leonurine treatment (Fig. 6A-B). $\mathrm{HE}$ staining of renal tissue further indicated the protective effect of leonurine on kidney damage. The severe tubular dilatation, marked tubular epithelial cell edema, and obvious cast formation induced by cisplatin were inhibited by leonurine treatment (Fig. 6C-D). In addition, the levels of two other known tubule damage biomarkers, KIM-1 and NGAL were elevated by cisplatin injection and reduced by leonurine treatment (Fig. $6 \mathrm{E}-\mathrm{F})$. Macrophages have been shown to be the primary cause of inflammatory infiltration in AKI. F4/80 staining was used to further evaluate the macrophage infiltration in cisplatin-challenged kidneys. As shown in Fig. 6G, the number of F4/80-positive cells was dramatically increased in the cisplatin treatment group but significantly decreased in the leonurine treatment group.

\subsection{Leonurine alleviates iron accumulation in CI-AKI}

Based on the critical ability of iron to initiate ferroptosis, we next assessed the changes in renal iron deposition. Compared to the control, cisplatin induced iron deposition, and both low-dose and high-dose leonurine significantly alleviated the increase in the iron deposition (Fig. 7A). We next found that the renal iron levels were increased by cisplatin treatment but reversed by leonurine treatment (Fig. 7B). The expression level of TFR, a membrane protein affecting iron transport, was significantly increased in the model group and inhibited by leonurine treatment. Moreover, the expression levels of FTH-1 and FTL were upregulated in cisplatin-treated mice but downregulated by leonurine treatment (Fig. 7C-D). Similar to the protein trends, the mRNA levels of TFR and FTH-1 were increased after cisplatin administration and decreased after leonurine treatment (Fig. 7E-F).

\subsection{Leonurine suppresses lipid peroxidation and ferroptosis in CI-AKI}

Next, we assessed the effects of leonurine on cisplatin-induced lipid peroxidation and ferroptosis in vivo. Compared to the control, cisplatin significantly increased the MDA content and decreased the GSH and SOD contents in the kidney, while leonurine obviously reversed the above changes (Fig. 8A-C). In addition, the protein levels of GPX4 and xCT were significantly downregulated by cisplatin treatment but dose-dependently restored by leonurine treatment. Moreover, the protein levels of Nrf2 and its regulated downstream antioxidases NQO1 and HO-1 were activated by leonurine treatment (Fig. 8D-F). Similar to the protein levels, the mRNA levels of GPX4 and $\mathrm{xCT}$ were decreased in the renal tissues of the model group. Interestingly, leonurine treatment significantly increased the transcript levels of GPX4 and xCT (Fig. 8G-H). Moreover, electron microscopic observations revealed mitochondrial damage in renal tissue cells of the model group, manifested as mitochondrial rupture and disappearance of mitochondrial cristae, and leonurine treatment significantly alleviated this damage (Fig. 8I).

\subsection{The renal protective effect of leonurine on CI-AKI is dependent on Nrf2 in mice}

We further evaluated whether the protective effect of leonurine on CI-AKI was dependent on Nrf2 using Nrf2 KO mice. As shown in Fig. 9A and B, leonurine significantly decreased the cisplatin-induced upregulation of SCr and BUN in the sera of wild-type mice, but these effects were nearly abolished in Nrf2 KO mice. Renal HE staining and protein expression of KIM1 and NGAL further demonstrated that the protective effect of leonurine on kidney damage was almost abrogated in Nrf2 KO mice (Fig. 9C-E, G). Moreover, the expression levels of Nrf2 and the downstream regulatory factors HO-1 and NQO1 were obviously increased in wild-type mice but not in Nrf2 KO mice (Fig. 9E-F). 


\subsection{Nrf2 deficiency prohibits the regulatory effect of leonurine on ferroptosis}

We further investigated whether the ability of leonurine to inhibit ferroptosis, as well as its cytoprotective potential, was dependent on Nrf2. In Nrf2 KO mice, cisplatin significantly increased the Fe and MDA levels and decreased the GSH levels compared to those in the wild-type mice (Fig. 10A-C). Moreover, treatment with leonurine markedly reversed the above changes in wild-type mice, while this effect was almost abolished in the Nrf2 KO mice. Moreover, the alleviating effects of leonurine on FTH-1 and TFR were eliminated (Fig. 10D-E). The protective effects of leonurine on the cisplatin-induced expression of the ferroptosis-related proteins GPX4 and xCT were also abolished in Nrf2 KO mice (Fig. 10D-F). DAB staining indicated that the reduced iron deposition in the leonurine treatment group was restored by the KO of Nrf2 (Fig. 10G). Furthermore, electron microscopy analysis revealed that the mitochondrial damage mitigated by leonurine was reversed in Nrf2 KO mice (Fig. 10H).

\section{Discussion and conclusions}

Ferroptosis and its related components are now better understood, and Nrf2 has been shown to play a key role in mediating this process. In particular, the antioxidant, iron, and intermediate metabolic statuses of cells are mediated by Nrf2 target genes. While the mechanisms of Nrf2 and ferroptosis in cisplatin-induced AKI have been elucidated, the relationship between them remains unclear(Hu et al., 2020; Ikeda et al., 2021; Shelton et al., 2013). In this study, we aimed to determine whether the nuclear transcription factor Nrf2, a critical regulator of the cellular antioxidant response, could inhibit cisplatin-induced ferroptosis in subjects with kidney injury. Moreover, we evaluated whether leonurine, an Nrf2 activator, could alleviate cisplatin-induced kidney injury by inhibiting Nrf2-mediated lipid peroxidation and ferroptosis.

Iron has been reported to play a key role in cisplatin-induced nephrotoxicity both in vitro and in vivo (Baliga et al., 1998). The abnormal accumulation of iron produces large amounts of free radicals that damage DNA, proteins, and other biomolecules (Stoyanovsky et al., 2019). Nrf2 is a key factor regulating the cellular antioxidant response, as it controls the expression of HO-1, NQO1 and other genes related to antioxidant stress. In addition to its important role in maintaining cellular redox balance, Nrf2 helps to mediate lipid peroxidation and ferroptosis (Abdalkader et al., 2018). The injection of cisplatin significantly increased lipid peroxidation and ROS production, which were reversed by the activation of Nrf2 or its downstream target genes. Notably, increased lipid oxidation and downstream Nrf2 target inactivation significantly enhance the overall protein lipid oxidation and ferroptosis in disease environments with low Nrf2, further promoting disease progression (La Rosa et al., 2021). However, whether Nrf2 levels are directly related to ferroptosis sensitivity in cisplatin-induced AKI has not been clarified. To elucidate the effect of Nrf2 on ferroptosis sensitivity in CI-AKI, we analyzed the iron accumulation, lipid peroxidation and expression of ferroptosisrelated proteins in cisplatin-treated wild-type and Nrf2 KO mice. Both the wild-type and Nrf2 KO mice exhibited altered iron accumulation, upregulated MDA levels, and downregulated protein levels of GPX4 and $\mathrm{xCT}$, which are biomarkers of ferroptosis. However, the abovementioned biomarkers were altered more significantly in Nrf2 KO mice than in the wild-type mice (Figs. 1-2). Therefore, we propose for the first time that the inhibition of Nrf2 aggravates ferroptosis and further enhances the progression of CI-AKI.

Considering the mitigated effect of Nrf2 on ferroptosis, targeting the upstream regulators of the ferroptotic cascade, including dysregulated iron levels and ROS production, by pharmacologically modulating the Nrf2 signaling pathway remains one of the best strategies for treating ferroptosis-related pathologies. We found that leonurine, an Nrf2 activator, significantly increased the cell viability and decreased the iron accumulation, ROS and cellular lipid ROS induced by erastin and RSL3 (Fig. 3). Iron is an essential element that is regulated by a variety of proteins. In general, iron is loaded onto transferrin, which binds to transferrin receptor 1 (TFR) on the cytoplasmic membrane and delivers iron to numerous tissues via endocytosis (Torti and Torti, 2013). Excess iron is stored in the protein ferritin, which is composed of 24 subunits of FTH-1 and FTL. Here, leonurine significantly reduced the protein expression levels of TFR, FTH-1 and FTL induced by RSL3 (Fig. 3). Next, we measured the levels of several other well-established biomarkers of ferroptosis, including GSH and lipid peroxidase-derived MDA, as well as those of GPX4 and xCT. As GSH depletion can trigger ferroptosis and MDA is the end-product of ferroptosis, we herein showed that leonurine inhibited the 
GSH depletion induced by RSL3 and the upregulation of MDA. Importantly, $\mathrm{xCT}$ and its key component Slc7a11 are responsible for the generation of intracellular GSH in response to oxidative stress. Inhibition of $\mathrm{xCT}$ reduces the level of GSH and the activity of glutathione peroxidase 4 (GPX4), thereby increasing lipid peroxidation. GPX4 deficiency is considered to be a biomarker of ferroptosis, and the depletion of GPX4 induces the ferroptosis of numerous renal tubular epithelial cells (Friedmann Angeli et al., 2014) (Seibt et al., 2019). In the present study, the downregulation of xCT and GPX4 induced by RSL3 was reversed by leonurine treatment in a dose-dependent manner (Fig. 4). Based on the ability of Nrf2 to prevent lipid peroxidation and ferroptosis, we hypothesized that the inhibitory effect of leonurine on ferroptosis is mediated by Nrf2. We further showed that the siRNA-mediated knockdown of Nrf2 ameliorated the effects of leonurine on the ROS production, lipid peroxidation, cellular iron level and expression of ferroptosis-related proteins induced by RSL3 (Fig. 5). In summary, our data showed that leonurine significantly activated Nrf2 and inhibited the ferroptosis of HK-2 cells induced by RSL3. The above effects were not observed after Nrf2 knockdown, which indicates that the protective effect of leonurine on ferroptosis in vitro may be mediated via the Nrf2 pathway.

As reported, leonurine is a therapeutic candidate for LPS-induced AKI and renal fibrosis (Cheng et al., 2015; Xu et al., 2014). However, the potential effects of leonurine on cisplatin-induced AKI have not yet been elucidated. Our results showed that leonurine significantly decreased the serum levels of Bun and SCr induced by cisplatin as well as the levels of KIM1 and NGAL and the histological extent of kidney injury (Fig. 6). Considering the abovementioned inhibitory effect of leonurine on ferroptosis induced by RSL3 in vitro, we further examined its effects on related ferroptosis biomarkers in the kidney. Consistent with the in vitro results, leonurine significantly inhibited the cisplatin-induced increases in iron accumulation and the TFR, FTL and FTH-1 levels in the kidney (Fig. 7). Furthermore, leonurine inhibited morphological and biochemical changes in factors related to ferroptosis, such as MDA, SOD and GSH depletion and the downregulation of GPX4 and xCT in cisplatin-induced AKI. In addition, leonurine markedly upregulated the expression of Nrf2, HO-1 and NQO1 (Fig. 8). Given that Nrf2 is a principal regulator of antioxidant responses that suppresses ferroptosis, we further investigated the potential for Nrf2 deficiency to ameliorate the protective effects of leonurine against cisplatin-induced ferroptosis. We treated Nrf2 KO mice with leonurine and then observed the histological and molecular parameters of cisplatin-induced AKI. The treatment of Nrf2 KO mice with leonurine did not rescue cisplatin-induced renal damage, as determined by the serum levels of Bun and Cre, the levels of KIM1 and NGAL, and the histological extent of kidney injury (Fig. 9). Furthermore, the treatment of Nrf2 KO mice with leonurine did not inhibit ferroptosis-related morphological or biochemical changes in cisplatin-induced AKI, as reflected by the MDA levels, SOD and GSH depletion, and downregulation of GPX4 and xCT (Fig. 10). Taken together, our results support that leonurine provides renal protection predominantly by activating the Nrf2-mediated inhibition of ferroptosis.

In conclusion, our study revealed the substantial significance of upregulating Nrf2 to prevent ferroptosis, thereby alleviating CI-AKI. This conclusion is derived from three key findings. First, Nrf2 KO mice were more susceptible to cisplatin-induced renal injury and ferroptosis. Second, the protective effect of leonurine against cisplatin-induced AKI was achieved by activating the antioxidant signaling molecule Nrf2 and inhibiting ferroptosis-related morphological and biochemical changes. Finally, the treatment of Nrf2 KO mice and Nrf2 siRNA HK-2 cells with leonurine nearly failed to rescue cisplatin- and RSL3-induced ferroptosis and renal injury in vivo and in vitro. The present study furthers our understanding of the mechanism by which Nrf2 inhibits ferroptosis and provides a strategy for investigating the antiferroptotic activity of Nrf2 activators in the context of cisplatin-induced AKI.

\section{Acknowledgments}

The study was supported by grants from the National Natural Science Foundation of China (Grant No. 81970576) and the Natural Science Foundation of Jilin (No. 20200201378JC and No. JLSCZD2019-065).

\section{Author contributions}

Xinxin Ci designed the study, Jianqiang $\mathrm{Hu}$, Wenjing Gu, Ning Ma and Xiaoye Fan carried out experiments 
and analyzed the data; Jianqiang $\mathrm{Hu}$ and Wenjing Gu drafted and revised the paper. The final version of the paper has been approved by all authors.

\section{Conflicts of interests}

All authors declare no competing interests.

\section{References:}

Abdalkader M., Lampinen R., Kanninen K. M., Malm T. M. and Liddell J. R. (2018). Targeting Nrf2 to Suppress Ferroptosis and Mitochondrial Dysfunction in Neurodegeneration. Front Neurosci, 12,466. doi: 10.3389/fnins.2018.00466.

Baliga R., Zhang Z., Baliga M., Ueda N. and Shah S. V. (1998). In vitro and in vivo evidence suggesting a role for iron in cisplatin-induced nephrotoxicity. Kidney Int, 53,394-401. doi: 10.1046/j.1523-1755.1998.00767.x.

Chen P., Chen F. and Zhou B. H. (2019). Leonurine ameliorates D-galactose-induced aging in mice through activation of the Nrf2 signalling pathway. Aging (Albany NY), 11,7339-7356. doi: 10.18632/aging.101733.

Cheng H., Bo Y., Shen W., Tan J., Jia Z., Xu C. and Li F. (2015). Leonurine ameliorates kidney fibrosis via suppressing TGF-beta and NF-kappaB signaling pathway in UUO mice. Int Immunopharmacol,25,406-415. doi: 10.1016/j.intimp.2015.02.023.

Deng F., Sharma I., Dai Y., Yang M. and Kanwar Y. S. (2019). Myo-inositol oxygenase expression profile modulates pathogenic ferroptosis in the renal proximal tubule. J Clin Invest,129,5033-5049. doi: 10.1172/JCI129903.

Deng F., Zheng X., Sharma I., Dai Y., Wang Y. and Kanwar Y. S. (2021). Regulated cell death in cisplatininduced AKI: relevance of myo-inositol metabolism. Am J Physiol Renal Physiol, 320,F578-F595. doi: 10.1152/ajprenal.00016.2021.

Dixon S. J. and Stockwell B. R. (2014). The role of iron and reactive oxygen species in cell death. Nat Chem Biol, 10,9-17. doi: 10.1038/nchembio.1416.

Dodson M., Castro-Portuguez R. and Zhang D. D. (2019). NRF2 plays a critical role in mitigating lipid peroxidation and ferroptosis.Redox Biol, 23,101107. doi: 10.1016/j.redox.2019.101107.

Fan X., Wei W., Huang J., Peng L. and Ci X. (2020). Daphnetin Attenuated Cisplatin-Induced Acute Nephrotoxicity With Enhancing Antitumor Activity of Cisplatin by Upregulating SIRT1/SIRT6-Nrf2 Pathway. Front Pharmacol, 11,579178. doi: 10.3389/fphar.2020.579178.

Friedmann Angeli J. P., Schneider M., Proneth B., Tyurina Y. Y., Tyurin V. A., Hammond V. J., Herbach N., Aichler M., Walch A., Eggenhofer E., Basavarajappa D., Radmark O., Kobayashi S., Seibt T., Beck H., Neff F., Esposito I., Wanke R., Forster H., Yefremova O., Heinrichmeyer M., Bornkamm G. W., Geissler E. K., Thomas S. B., Stockwell B. R., O'Donnell V. B., Kagan V. E., Schick J. A. and Conrad M. (2014). Inactivation of the ferroptosis regulator Gpx4 triggers acute renal failure in mice. Nat Cell Biol, 16,1180-1191. doi: $10.1038 /$ ncb3064.

Herzog C., Yang C., Holmes A. and Kaushal G. P. (2012). zVAD-fmk prevents cisplatin-induced cleavage of autophagy proteins but impairs autophagic flux and worsens renal function. Am J Physiol Renal Physiol, 303,F1239-1250. doi: 10.1152/ajprenal.00659.2011.

Hoste E. A. J., Kellum J. A., Selby N. M., Zarbock A., Palevsky P. M., Bagshaw S. M., Goldstein S. L., Cerda J. and Chawla L. S. (2018). Global epidemiology and outcomes of acute kidney injury. Nat Rev Nephrol, 14,607-625. doi: 10.1038/s41581-018-0052-0.

Hu Z., Zhang H., Yi B., Yang S., Liu J., Hu J., Wang J., Cao K. and Zhang W. (2020). VDR activation attenuate cisplatin induced AKI by inhibiting ferroptosis. Cell Death Dis, 11,73. doi: 10.1038/s41419-020$2256-\mathrm{z}$. 
Ikeda Y., Hamano H., Horinouchi Y., Miyamoto L., Hirayama T., Nagasawa H., Tamaki T. and Tsuchiya K. (2021). Role of ferroptosis in cisplatin-induced acute nephrotoxicity in mice. J Trace Elem Med Biol, 67,126798. doi: 10.1016/j.jtemb.2021.126798.

La Rosa P., Petrillo S., Turchi R., Berardinelli F., Schirinzi T., Vasco G., Lettieri-Barbato D., Fiorenza M. T., Bertini E. S., Aquilano K. and Piemonte F. (2021). The Nrf2 induction prevents ferroptosis in Friedreich's Ataxia. Redox Biol, 38,101791. doi: 10.1016/j.redox.2020.101791.

Li Y. Y., Lin Y. K., Liu X. H., Wang L., Yu M., Li D. J., Zhu Y. Z. and Du M. R. (2020). Leonurine: From Gynecologic Medicine to Pleiotropic Agent. Chin J Integr Med, 26,152-160. doi: 10.1007/s11655-019-3453-0.

Linkermann A., Chen G., Dong G., Kunzendorf U., Krautwald S. and Dong Z. (2014). Regulated cell death in AKI. J Am Soc Nephrol,25,2689-2701. doi: 10.1681/ASN.2014030262.

Maiorino M., Conrad M. and Ursini F. (2018). GPx4, Lipid Peroxidation, and Cell Death: Discoveries, Rediscoveries, and Open Issues.Antioxid Redox Signal, 29,61-74. doi: 10.1089/ars.2017.7115.

Park E. and Chung S. W. (2019). ROS-mediated autophagy increases intracellular iron levels and ferroptosis by ferritin and transferrin receptor regulation. Cell Death Dis, 10,822. doi: 10.1038/s41419-019-2064-5.

Seibt T. M., Proneth B. and Conrad M. (2019). Role of GPX4 in ferroptosis and its pharmacological implication. Free Radic Biol Med, 133,144-152. doi: 10.1016/j.freeradbiomed.2018.09.014.

Shelton L. M., Park B. K. and Copple I. M. (2013). Role of Nrf2 in protection against acute kidney injury. Kidney Int, 84,1090-1095. doi: 10.1038/ki.2013.248.

Stockwell B. R., Friedmann Angeli J. P., Bayir H., Bush A. I., Conrad M., Dixon S. J., Fulda S., Gascon S., Hatzios S. K., Kagan V. E., Noel K., Jiang X., Linkermann A., Murphy M. E., Overholtzer M., Oyagi A., Pagnussat G. C., Park J., Ran Q., Rosenfeld C. S., Salnikow K., Tang D., Torti F. M., Torti S. V., Toyokuni S., Woerpel K. A. and Zhang D. D. (2017). Ferroptosis: A Regulated Cell Death Nexus Linking Metabolism, Redox Biology, and Disease. Cell, 171,273-285. doi: 10.1016/j.cell.2017.09.021.

Stoyanovsky D. A., Tyurina Y. Y., Shrivastava I., Bahar I., Tyurin V. A., Protchenko O., Jadhav S., Bolevich S. B., Kozlov A. V., Vladimirov Y. A., Shvedova A. A., Philpott C. C., Bayir H. and Kagan V. E. (2019). Iron catalysis of lipid peroxidation in ferroptosis: Regulated enzymatic or random free radical reaction? Free Radic Biol Med,133,153-161. doi: 10.1016/j.freeradbiomed.2018.09.008.

Torti S. V. and Torti F. M. (2013). Iron and cancer: more ore to be mined. Nat Rev Cancer, 13,342-355. doi: $10.1038 / \operatorname{nrc} 3495$.

Tristao V. R., Goncalves P. F., Dalboni M. A., Batista M. C., Durao Mde S., Jr. and Monte J. C. (2012). Nec-1 protects against nonapoptotic cell death in cisplatin-induced kidney injury. Ren Fail, 34,373-377. doi: 10.3109/0886022X.2011.647343.

Wang L., Liu Y., Du T., Yang H., Lei L., Guo M., Ding H. F., Zhang J., Wang H., Chen X. and Yan C. (2020). ATF3 promotes erastin-induced ferroptosis by suppressing system Xc(.). Cell Death Differ,27,662-675. doi: 10.1038/s41418-019-0380-z.

Xie Y. Z., Zhang X. J., Zhang C., Yang Y., He J. N. and Chen Y. X. (2019). Protective effects of leonurine against ischemic stroke in mice by activating nuclear factor erythroid 2-related factor 2 pathway. CNS Neurosci Ther, 25,1006-1017. doi: 10.1111/cns.13146.

Xu D., Chen M., Ren X., Ren X. and Wu Y. (2014). Leonurine ameliorates LPS-induced acute kidney injury via suppressing ROS-mediated NF-kappaB signaling pathway. Fitoterapia, 97,148-155. doi: 10.1016/j.fitote.2014.06.005.

Yang Y., Adebali O., Wu G., Selby C. P., Chiou Y. Y., Rashid N., Hu J., Hogenesch J. B. and Sancar A. (2018). Cisplatin-DNA adduct repair of transcribed genes is controlled by two circadian programs in mouse tissues. Proc Natl Acad Sci U S A, 115,E4777-E4785. doi: 10.1073/pnas.1804493115. 
Figure legends:

Fig. 1 Nrf2 KO mice exhibited more severe renal injury and iron metabolism disruption after cisplatin stimulation than wild-type mice.C57BL/6 mice with or without Nrf2 gene KO were treated by an intraperitoneal injection of cisplatin $(20 \mathrm{mg} / \mathrm{kg})$ or vehicle. Three days later, the mice were sacrificed, and their serum and renal tissues were collected. The serum BUN (A) and SCr (B) levels were then detected. (C) and (D) HE staining indicated renal tissue damage. (E) The serum contents of iron ions were measured to assess the iron metabolism in the mice. ( $\mathrm{F}$ to $\mathrm{H}$ ) The protein levels of Nrf2, NQO1, HO-1, FTH-1, FTL and TFR in renal tissues were analyzed by Western blot. All experiments were repeated 5 times, $\mathrm{P}^{*}$ [?] 0.05 and $\mathrm{P}^{* *}[?] 0.01$ versus the control group, $\mathrm{P}^{\#}$ [?] 0.05 and $\mathrm{P}^{\# \#}$ [?] 0.01 versus the WT CDDP group. WT, wild-type C57BL/6 mice; KO, Nrf2 KO C57BL/6 mice.

Fig. 2 Cisplatin-stimulated Nrf2 KO mice exhibited more severe lipid peroxidation than wildtype mice. Renal tissues were collected for the measurement of (A) MDA and (B) GSH. (C) and (D) The protein expression levels of GPX4 and $\mathrm{xCT}$ in renal tissues were analyzed by Western blot. (E) and (F) The mRNA levels of GPX4 and SLC7A11 in renal tissues were detected by real-time quantitative PCR. All experiments were repeated 5 times, $\mathrm{P}^{*}$ [?] 0.05 and $\mathrm{P}^{* *}$ [?] 0.01 versus the control group, $\mathrm{P}^{\#}$ [?] 0.05 and $\mathrm{P} \# \#$ [?] 0.01 versus the WT CDDP group.

Fig. 3 Leonurine protects HK-2 cells from ferroptosis. HK-2 cells were treated with gradient concentrations of erastin (A) or RSL3 (B) for $24 \mathrm{~h}$, and the cell survival rate was detected by the CCK-8 assay. (C) and (D) After $24 \mathrm{~h}$ of treatment with or without leonurine $(100 \mu \mathrm{M})$, HK-2 cells were stimulated with erastin $(30 \mu \mathrm{M})$ or RSL3 $(0.4 \mu \mathrm{M})$, and the cell survival rate was detected by the CCK-8 assay at $24 \mathrm{~h}$. (E) The cell iron content was then measured by calcein-AM staining and measurement of the OD value. (F) The above mentioned cells were stained with FerroOrange and detected by confocal microscopy. ( $\mathrm{G}$ and $\mathrm{H}$ ) The protein levels of TFR, FTL and FTH-1 in HK-2 cells were analyzed by Western blot. All experiments were repeated 5 times, $\mathrm{P}^{*}$ [?] 0.05 and $\mathrm{P}^{* *}$ [?] 0.01 versus the control group, $\mathrm{P}^{\#}$ [?] 0.05 and $\mathrm{P}^{\# \#[?]} 0.01$ versus the RSL3-treated group.

Fig. 4 Leonurine alleviates lipid peroxidation induced by RSL3 in HK-2 cells. (A) The ROS content in HK-2 cells was measured by DCFH-DA. (B) Liperfluo staining, as observed by confocal microscopy, was used to detect lipid peroxidation in HK-2 cells. HK-2 cells were harvested, and the levels of (C) GSH and (D) MDA were detected. (E to G) The protein levels of xCT, GPX4, Nrf2, NQO1 and HO-1 in HK-2 cells were analyzed by Western blot. All experiments were repeated 5 times, $\mathrm{P}^{*}$ [?] 0.05 and $\mathrm{P}^{* *}$ [?] 0.01 versus the control group, $\mathrm{P}^{\#}$ [?] 0.05 and $\mathrm{P}^{\# \#[?]} 0.01$ versus the RSL3-treated group.

Fig. 5 The inhibitory effect of leonurine on HK-2 cell ferroptosis is dependent on Nrf2 gene expression. (A and B) The efficiency of Nrf2 knockdown in HK-2 cells was analyzed by Western blot. (C) The CCK- 8 assay was performed to determine the survival rate of cells. The contents of (D) GSH and (E) MDA in HK-2 cells were detected by appropriate kits. (F) The ROS level in HK-2 cells was measured by DCFH-DA. (G) The iron content in HK-2 cells was detected by calcein-AM staining. (H to J) The protein levels of FTH-1, TFR, xCT and GPX4 in HK-2 cells were analyzed by Western blot. All experiments were repeated 5 times, $\mathrm{P}^{*}$ [?] 0.05 and $\mathrm{P}^{* *}$ [?] 0.01 versus the control group, $\mathrm{P}^{\#}$ [?] 0.05 and $\mathrm{P}^{\# \# ~[?] ~} 0.01$ versus the RSL3-treated group. NS, no specificity.

Fig. 6 Leonurine alleviates cisplatin-induced acute renal injury in vivo. The animals were grouped based on the treatment administered. On the fourth day, the mice were sacrificed, and their blood and kidneys were harvested. The levels of (A) BUN and (B) SCR in renal tissues were detected. (C-D) HE staining was performed to determine the degree of renal injury, and the renal injury indices were calculated according to the standard method. (E-F) The protein levels of KIM1 and NGAL in renal tissues were analyzed by Western blot. (G) F4/80 staining was performed to determine the degree of renal tissue inflammation. All experiments were repeated 5 times, $\mathrm{P}^{*}$ [?] 0.05 and $\mathrm{P}^{* *}$ [?] 0.01 versus the control group, $\mathrm{P}^{\#}$ [?] 0.05 and $\mathrm{P} \# \#$ [?] 0.01 versus the WT CDDP group.

Fig. 7 Leonurine reverses the cisplatin-induced iron metabolism disruption in the mouse 
kidney. (A) DAB staining was performed to detect the iron deposition in renal tissues. (B) The iron content in renal tissues was detected by an iron assay kit. (C and D) The protein levels of TFR, FTL and FTH-1 in renal tissues were analyzed by Western blot. (E and F) The mRNA levels of FTH-1 and TFR in renal tissues were measured by real-time quantitative PCR. All experiments were repeated 5 times, $\mathrm{P}^{*}[?]$ 0.05 and $\mathrm{P}^{* *}$ [?] 0.01 versus the control group, $\mathrm{P}^{\#}$ [?] 0.05 and $\mathrm{P}^{\# \# ~[?] ~} 0.01$ versus the WT CDDP group.

Fig. 8 Leonurine alleviates cisplatin-induced lipid peroxidation via the Nrf2 pathway. The levels of (A) MDA, (B) GSH and (C) SOD in renal tissues were detected by the appropriate assay kits. (D-F) The protein levels of GPX4, xCT, Nrf2, NQO1 and HO-1 in renal tissues were analyzed by Western blot. The mRNA levels of (G) GPX4 and (H) SLC7A11 in renal tissues were measured by real-time quantitative PCR. (I) The mitochondrial structure of renal cells was observed under an electron microscope. All experiments were repeated 5 times, $\mathrm{P}^{*}[?] \quad 0.05$ and $\mathrm{P}^{* *}$ [?] 0.01 versus the control group, $\mathrm{P}^{\#}$ [?] 0.05 and $\mathrm{P}^{\# \# ~[?]} 0.01$ versus the WT CDDP group.

Fig. 9 Leonurine alleviates cisplatin-induced renal injury in vivo, and this phenomenon is dependent on Nrf2 gene expression. C57BL/6 mice with or without Nrf2 gene KO were subjected to the same treatments as described for the abovementioned animal experiments. (A and B) The levels of BUN and $\mathrm{SCr}$ in mouse kidneys were detected. (C and D) HE staining was performed to detected changes in renal tissue damage based on the evaluation of renal injury criteria. (E to G) The protein levels of Nrf2, NQO1, HO-1, KIM1 and NGAL in renal tissues were analyzed by Western blot. All experiments were repeated 5 times, $\mathrm{P}^{*}$ [?] 0.05 and $\mathrm{P}^{* *}$ [?] 0.01 versus the control group, $\mathrm{P}^{\#}$ [?] 0.05 and $\mathrm{P}^{\# \#[?]} 0.01$ versus the WT CDDP group. NS, no specificity.

Fig. 10 The effect of leonurine on alleviating cisplatin-induced ferroptosis in vivo depends on Nrf2 gene expression. The levels of (A) $\mathrm{Fe}^{2+}$, (B) GSH and (C) MDA in renal tissues were measured by the appropriate assay kits. (D-F) The protein levels of FTH-1, TFR, GPX4 and xCT in renal tissues were analyzed by Western blot. $(\mathrm{G})$ The iron deposition in renal tissues was detected by DAB staining. $(\mathrm{H})$ Morphological changes in renal cell mitochondria were observed by electron microscopy. All experiments were repeated 5 times, $\mathrm{P}^{*}$ [?] 0.05 and $\mathrm{P}^{* *}$ [?] 0.01 versus the control group, $\mathrm{P}^{\#}$ [?] 0.05 and $\mathrm{P}^{\# \#[?] ~} 0.01$ versus the WT CDDP group, NS, no specificity.

Figures 

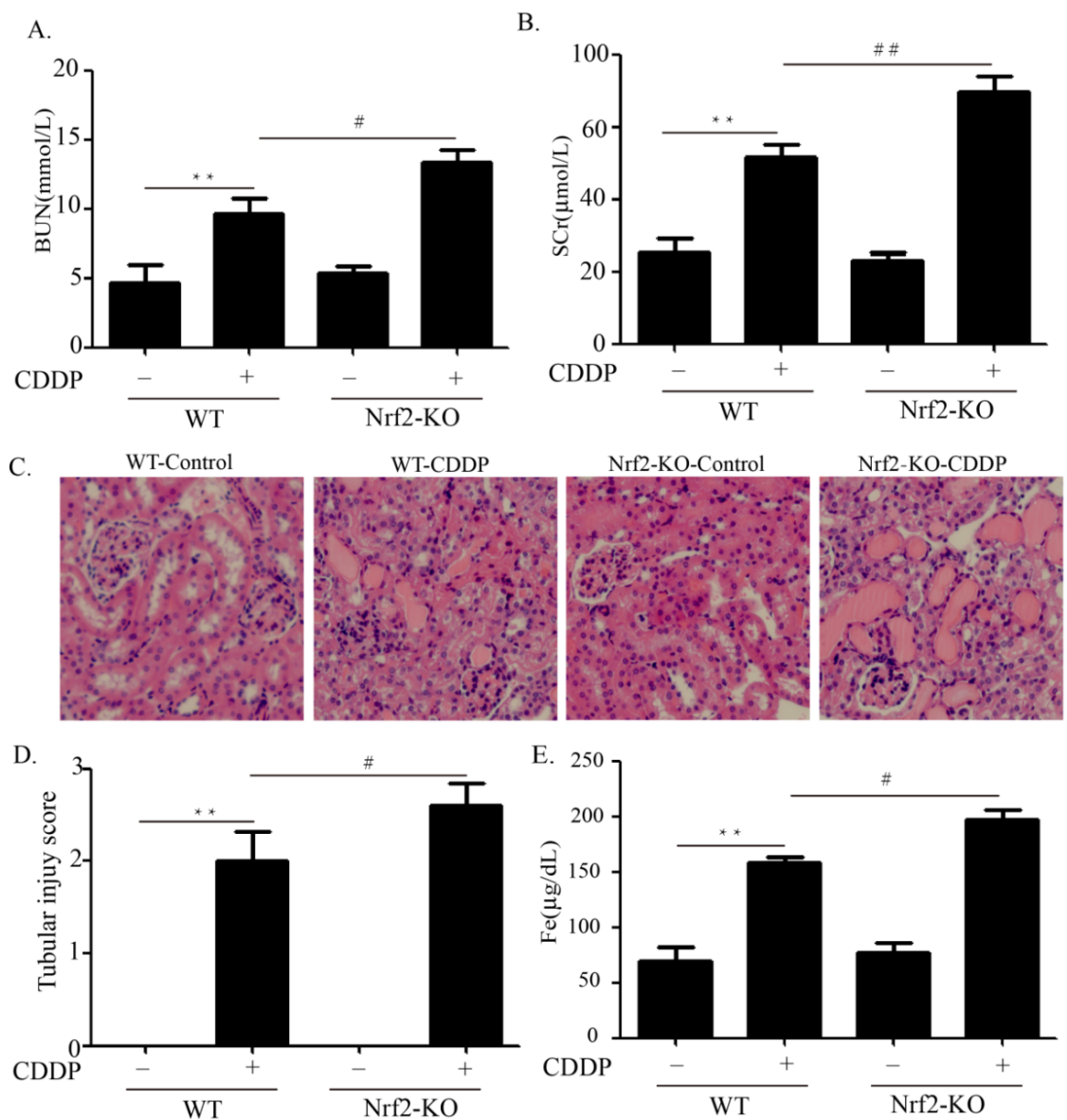

F.

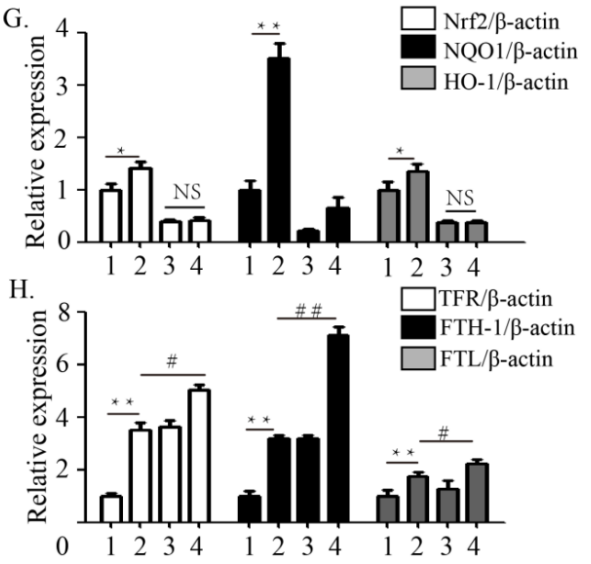

Figure 1 
A.

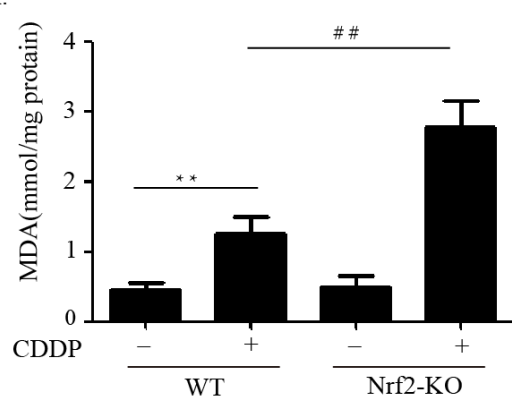

C.

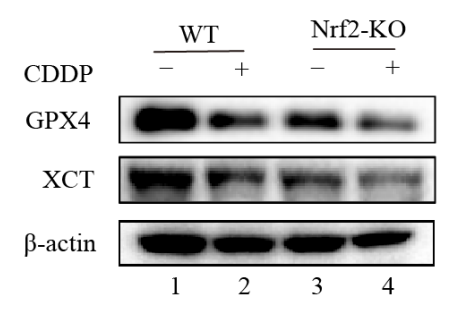

E.

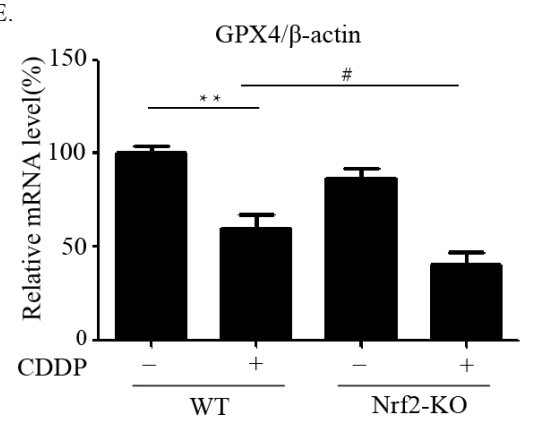

B.

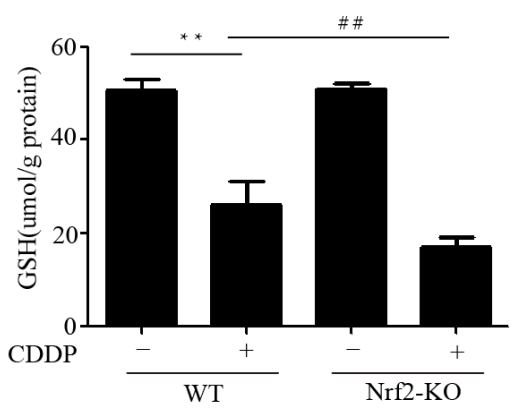

D.

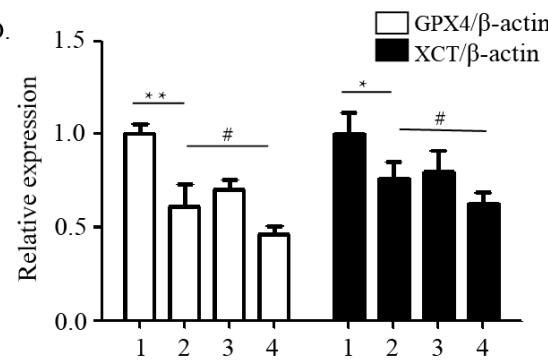

F.

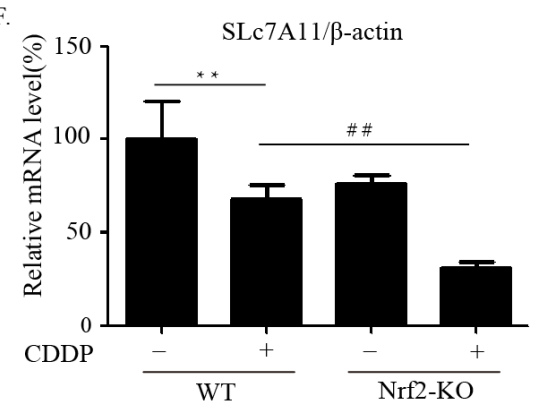

Figure 2 
A.

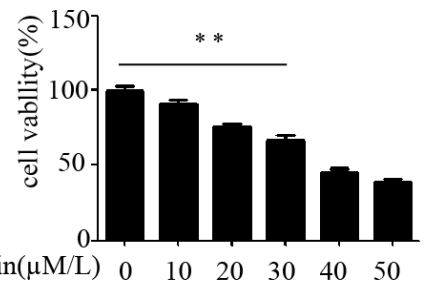

C.

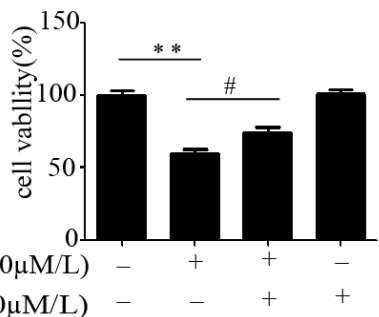

$\operatorname{Erastin}(30 \mu \mathrm{M} / \mathrm{L})$
$\operatorname{leo}(100 \mu \mathrm{M} / \mathrm{L})$

E.
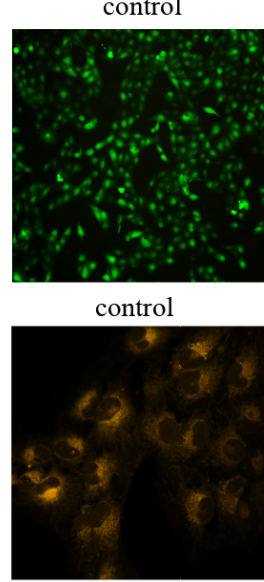

G.

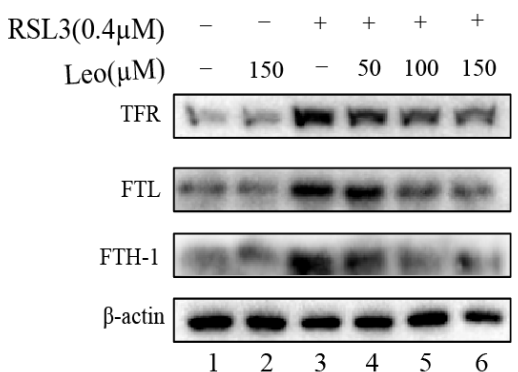

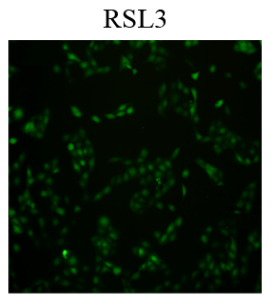

RSL3

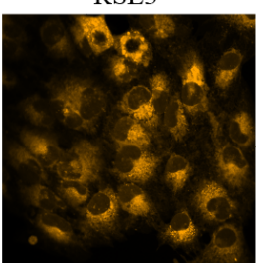

$\mathrm{H}$.
B.

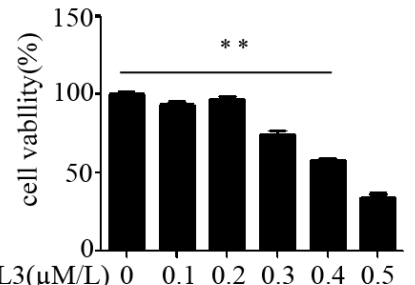

$\begin{array}{llllllll}\mathrm{RSL} 3(\mu \mathrm{M} / \mathrm{L}) & 0 & 0.1 & 0.2 & 0.3 & 0.4 & 0.5\end{array}$

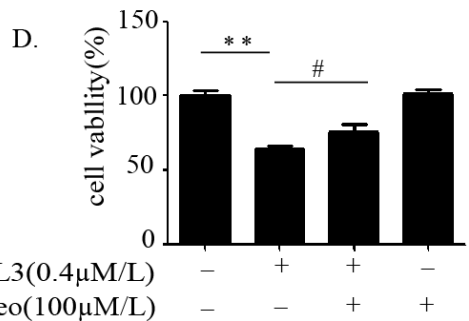

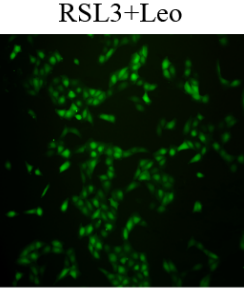

RSL3+Leo
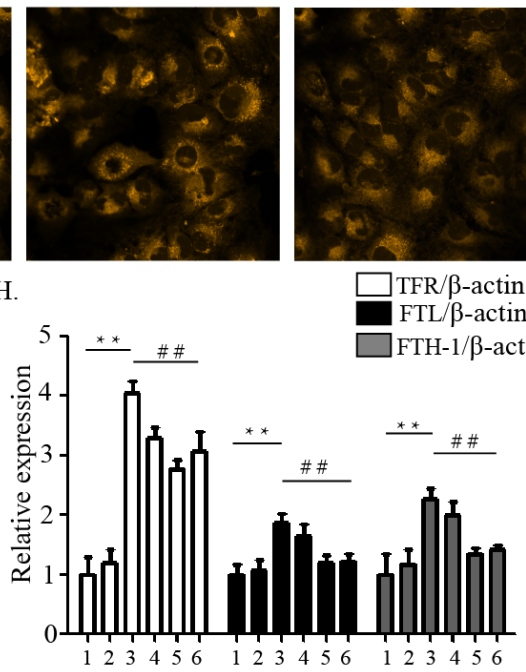

Figure 3 


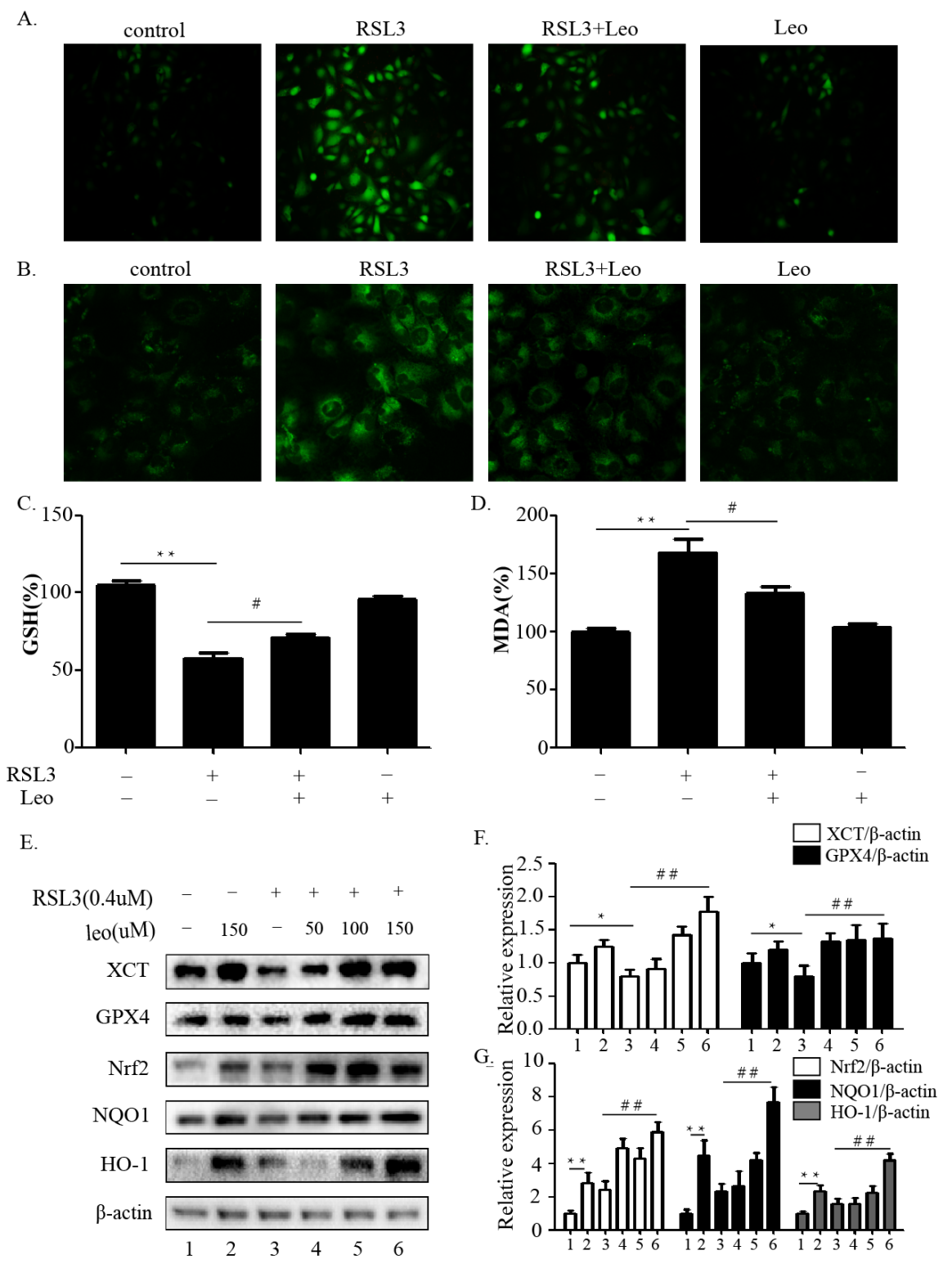

Figure 4 

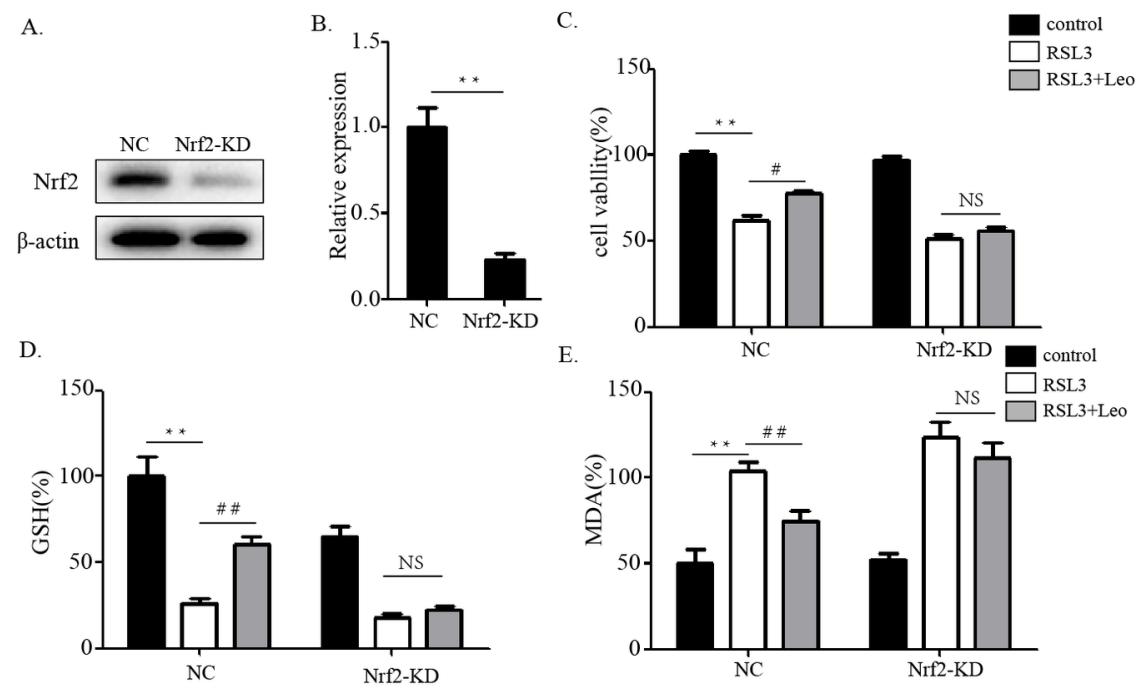

F.
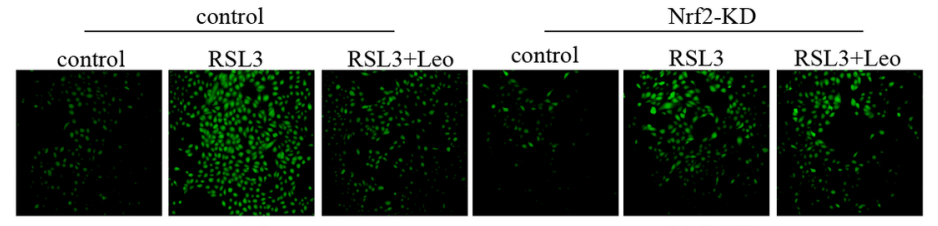

G.
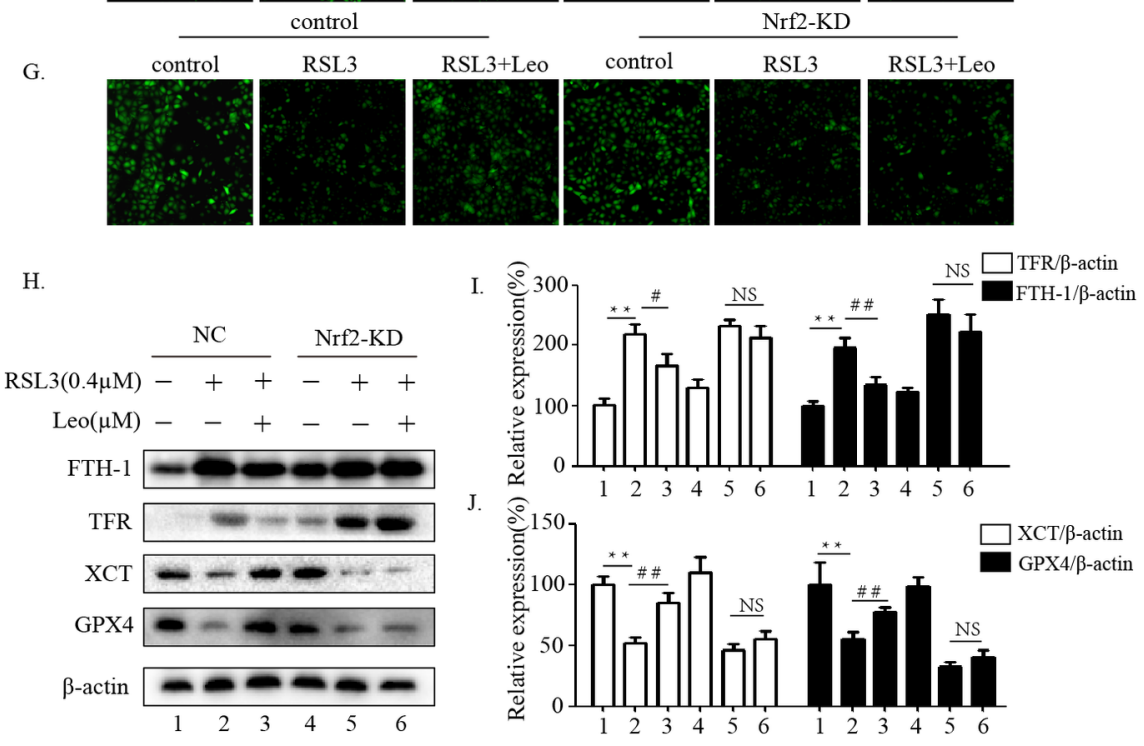

Figure 5 


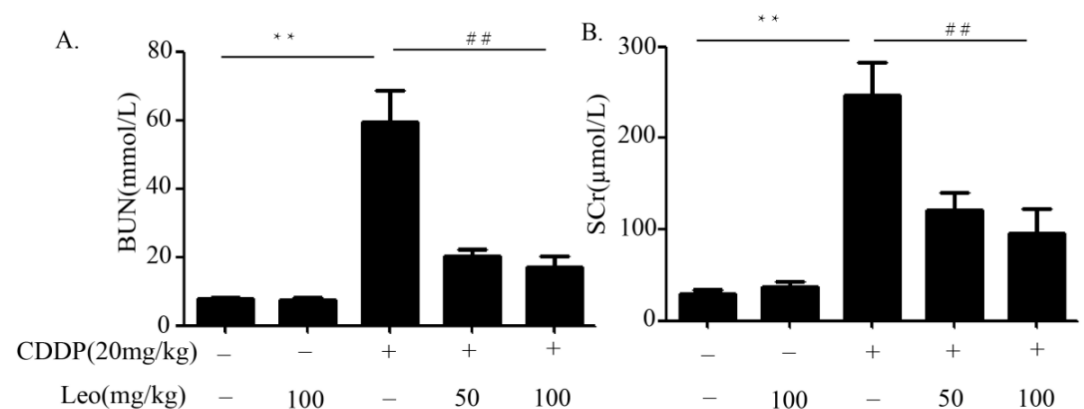

C.

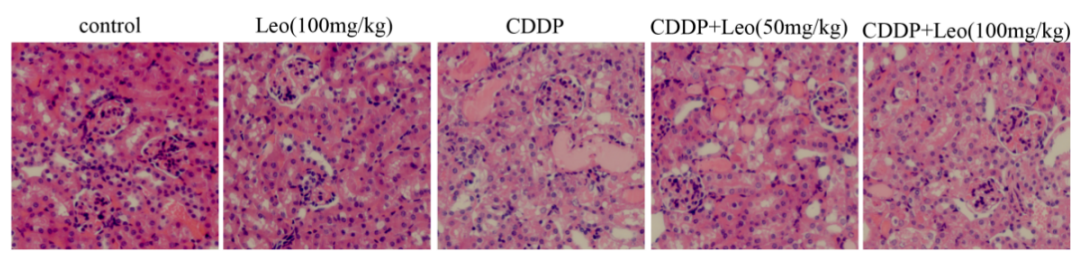

D.
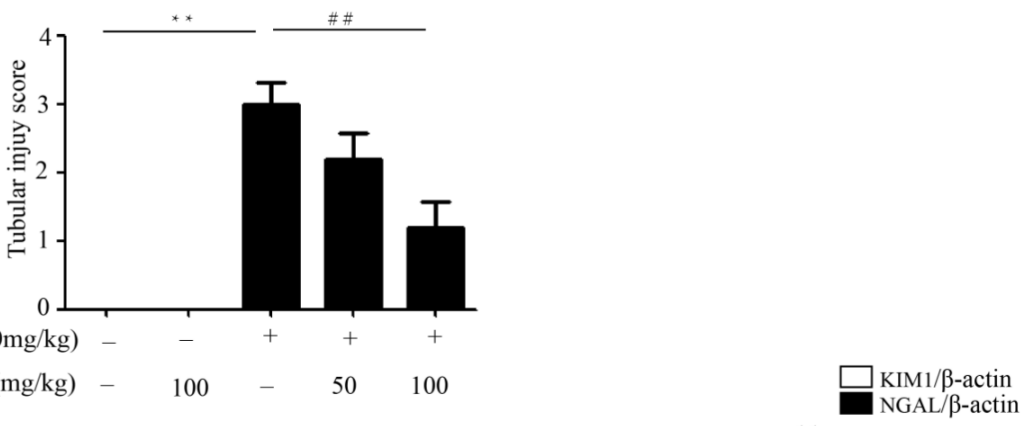

E.

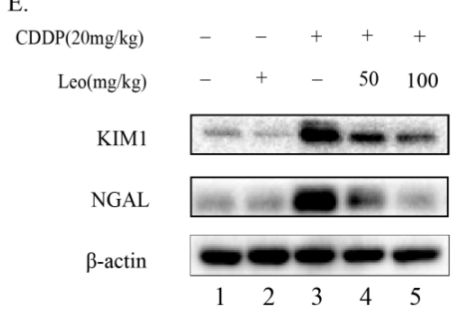

G

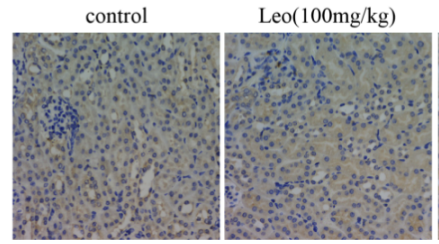

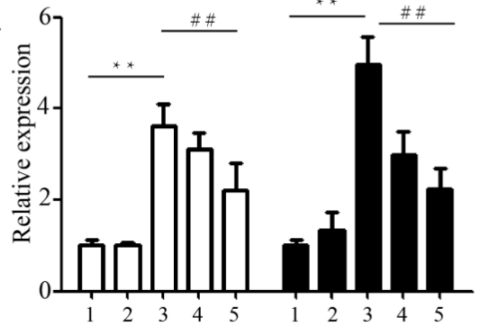

CDDP CDDP+Leo $(50 \mathrm{mg} / \mathrm{kg})$ CDDP+Leo $(100 \mathrm{mg} / \mathrm{kg})$
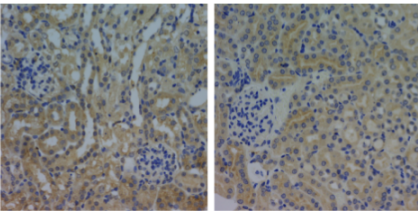

Figure 6 
A.

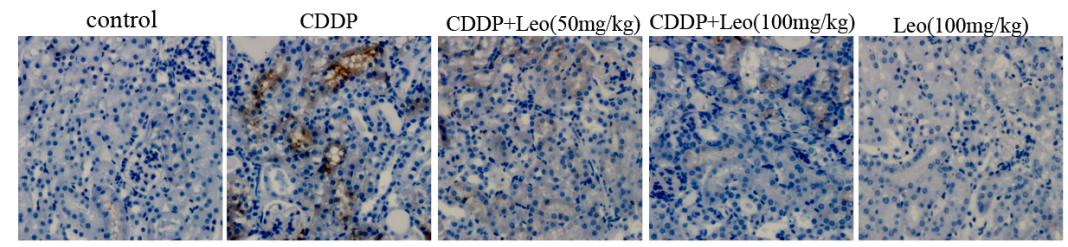

B.

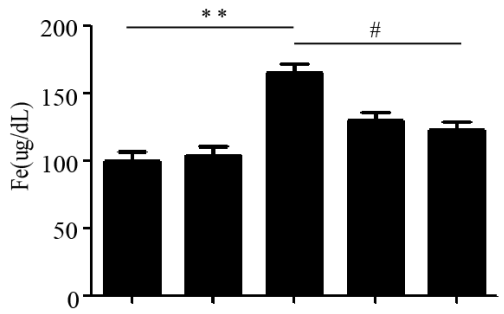

$\operatorname{CDDP}(20 \mathrm{mg} / \mathrm{kg})-$

Leo $(\mathrm{mg} / \mathrm{kg}) \quad-\quad 100 \quad-\quad 50 \quad 100$

C. $\operatorname{CDDP}(20 \mathrm{mg} / \mathrm{kg})+-\quad+\quad+\quad+\quad$ D.
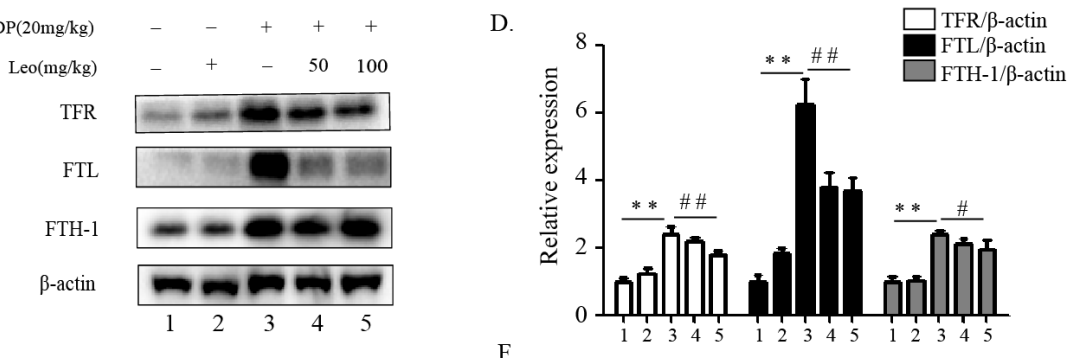

E.
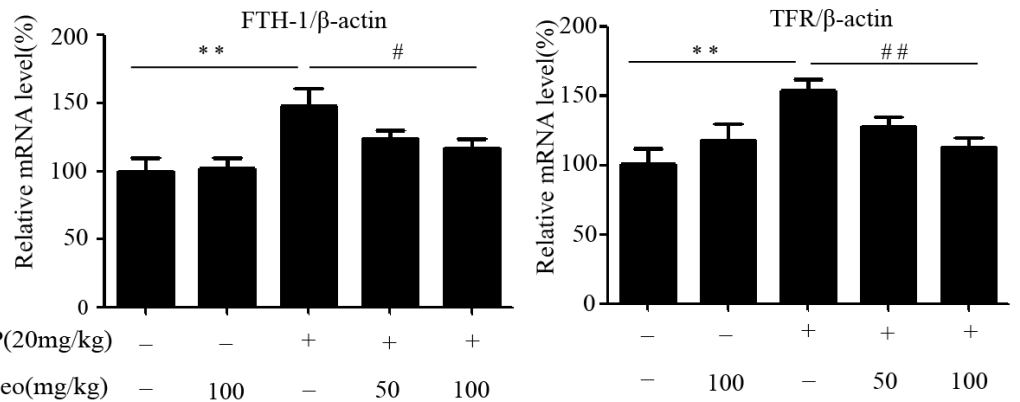

Figure 7 


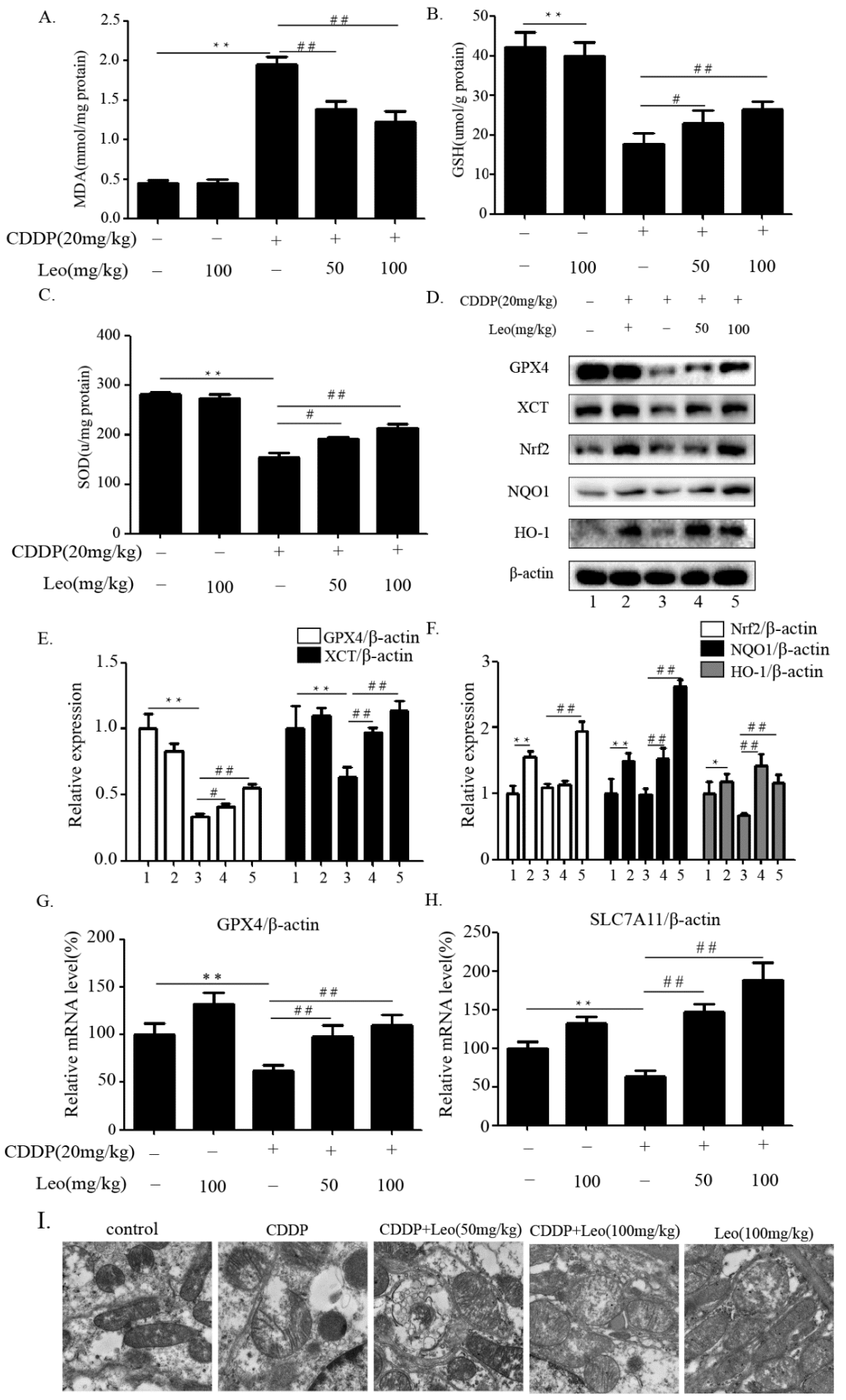

Figure 8 


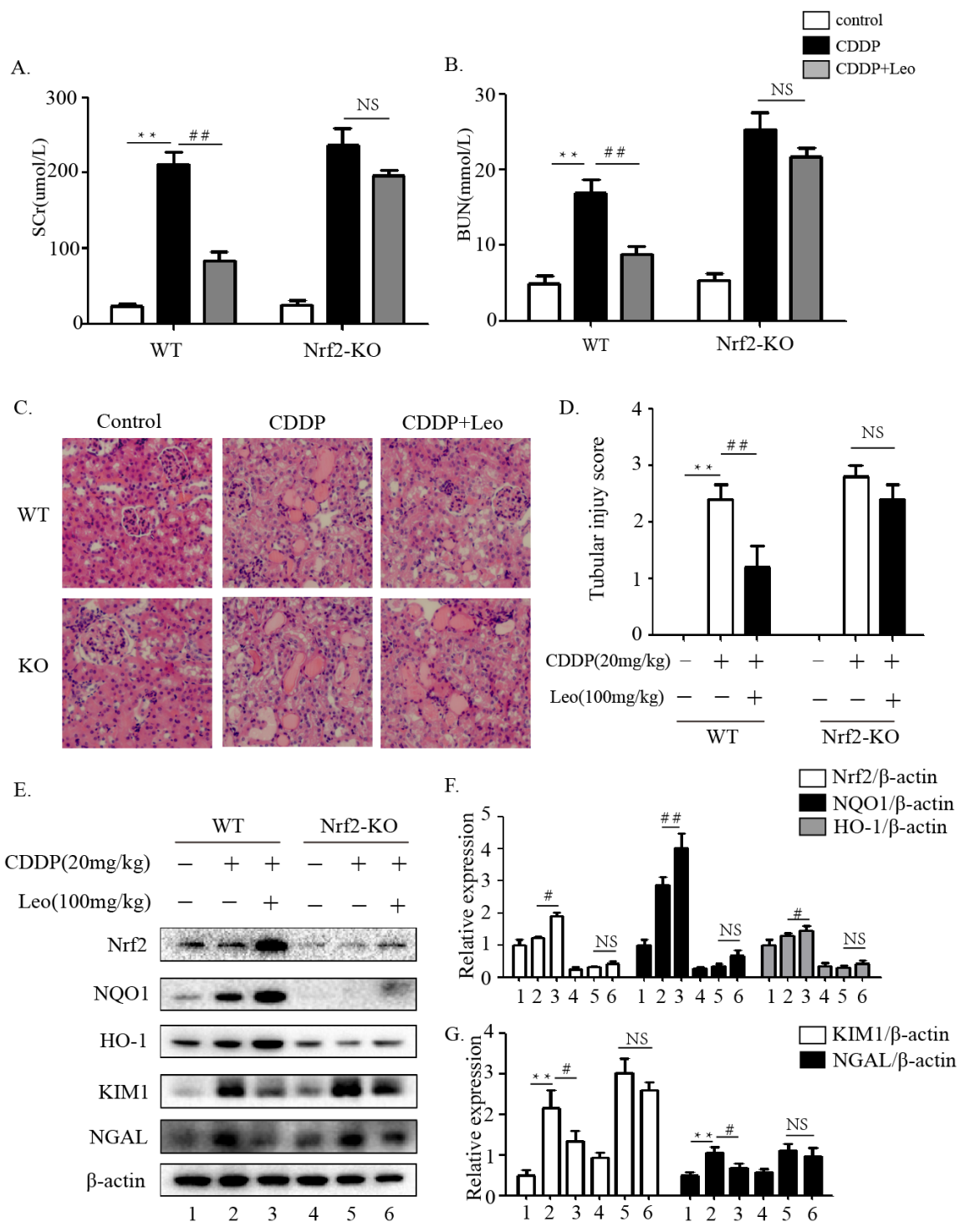

Figure 9 

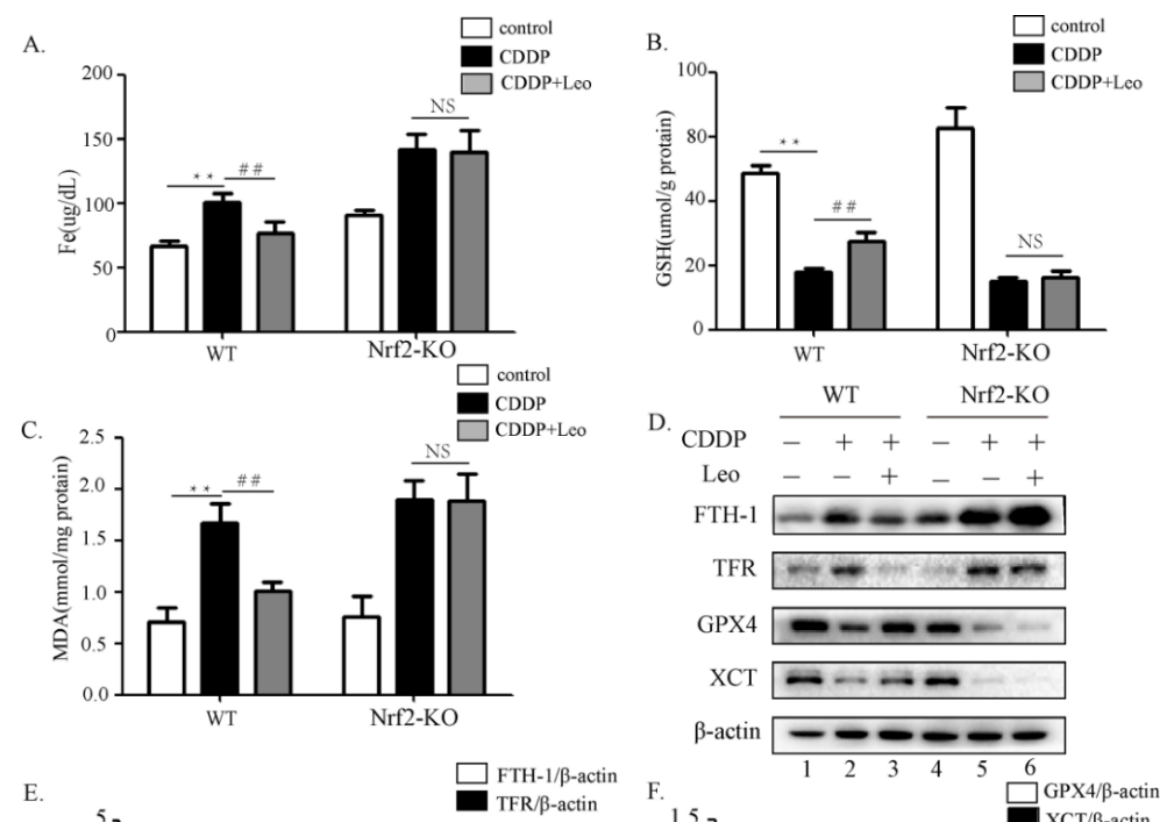

E.
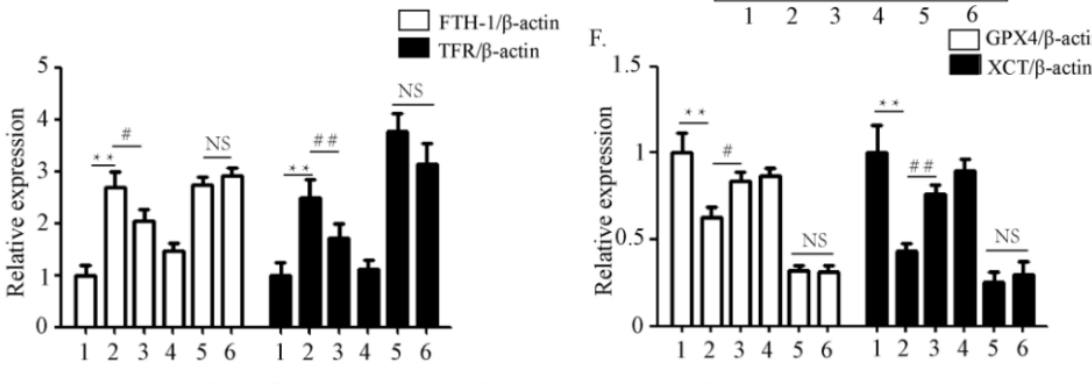

G
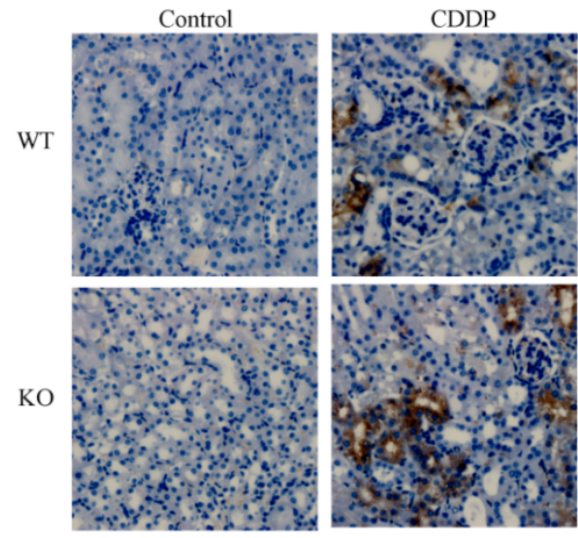

CDDP+Leo

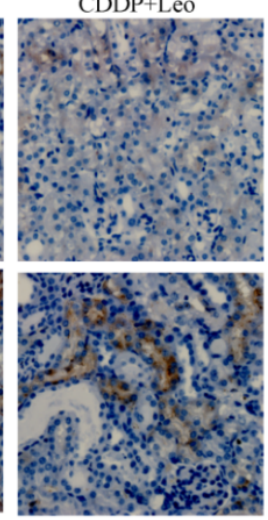

H.
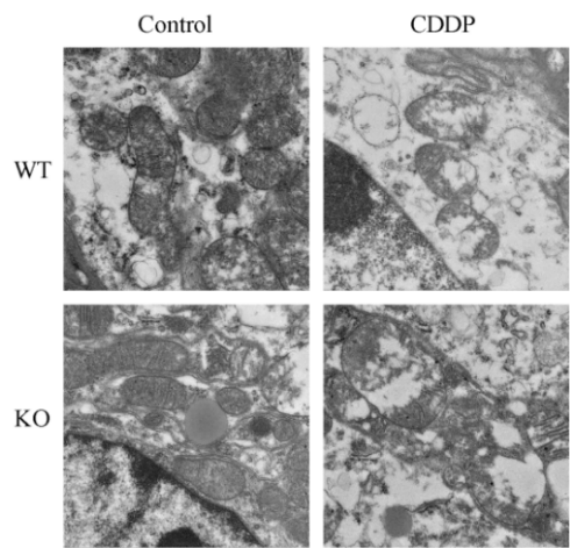

CDDP+Leo

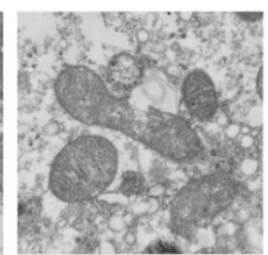


Fig. 10 